\title{
ANALYTIC GEOMETRY OF COMPLEX SUPERSPACES
}

\author{
H. FLENNER AND D. SUNDARARAMAN
}

\begin{abstract}
A detailed account of the analytic geometry of complex superspaces is given in this paper. Several representability criteria and representability theorems are proved. In particular, the existence of a versal family of deformations (parametrized by a complex superspace) for any compact complex superspace is proved.
\end{abstract}

\section{INTRODUCTION}

In recent years, complex analytic and algebraic geometric methods have been applied to several aspects of theoretical physics. Motivated by supersymmetry and second quantization, a branch of geometry called supergeometry is being actively studied by theoretical physicists. For introductions to supergeometry, one may refer to Berezin [Be], Leítes [L], and Manin $\left[\mathrm{Ma}_{1}, \mathrm{Ma}_{2}\right]$. The latter references also discuss the scope of supergeometry in mathematics, apart from its implications to physics.

Our interests in this paper are in the mathematical aspects of supergeometry; we give a detailed account of the analytic geometry of complex superspaces, from the point of view of deformations and moduli. In essence we prove several representability criteria and representability theorems. For the study of moduli, algebraic geometers have found that abstract functorial methods are not only quite convenient but also become indispensible. Our work relies very much on these functorial and algebraic methods. Artin's approximation theorems and the formal deformation (moduli) theory of Rim [Ri], Schlessinger [Sch], and Artin $\left[\mathrm{A}_{2}\right]$ are powerful abstract methods for solving concrete moduli problems in algebraic geometry. Their analogues in analytic geometry have been proved and applied by several mathematicians; especially relevant to our context are the works of Schuster [Schu], Bingener $\left[\mathrm{Bi}_{1}, \mathrm{Bi}_{2}\right]$ and the author $\left[\mathrm{F}_{1}, \mathrm{~F}_{2}, \mathrm{~F}_{3}\right]$. A major part of this work is devoted to generalizing these works to complex supergeometry.

We now outline the contents of this paper, after giving some basic facts and definitions of complex supergeometry.

Let $A=A_{0}+A_{1}$ be a $\mathbb{Z}_{2}$-graded associative ring. Elements of $A_{0}$ (resp. $A_{1}$ ) are said to have parity 0 (resp. 1). The parity of a homogeneous element $a_{\varepsilon}$, denoted by $\tilde{a}$, is $\varepsilon, \varepsilon \in\{0,1\}$. Elements of $A_{0}$ (resp. $\left.A_{1}\right)$ are called the even (resp. odd) elements of $A$. The supercommutator of two homogeneous

Received by the editors April 13, 1990.

1980 Mathematics Subject Classification (1985 Revision). Primary 14J15, 32J13; Secondary 14B12, 32G05. 
elements $a, b$ is defined to be the element $a b-(-1)^{\tilde{a} \tilde{b}} b a$ and is denoted by $[a, b]$. The supercommutator of any two elements of $A$ is defined by additivity. $a$ and $b$ are said to supercommute if their supercommutator is zero. The ring $A$ is said to be a supercommutative ring if any two of its elements supercommute. An analytic superalgebra is a superalgebra $A=A_{0}+A_{1}$, where $A_{0}$ is a convergent analytic algebra over $\mathbb{C}$ (i.e., $A_{0}$ is a quotient of a convergent power series ring over $\mathbb{C}$ ) and $A_{1}$ is a finite $A_{0}$-module.

A superspace is a ringed space $\left(X, \mathscr{O}_{X}\right)$, where $X$ is a Hausdorff topological space and $\mathscr{O}_{X}$ is a sheaf of local supercommutative rings on $X$. Thus, in particular the structure sheaf $\mathscr{O}_{X}$ is $\mathbb{Z}_{2}$-graded: $\mathscr{O}_{X}=\mathscr{O}_{X, 0} \oplus \mathscr{O}_{X, 1} \cdot\left(X, \mathscr{O}_{X, 0}\right)$ is called the even part and $\mathscr{O}_{X, 1}$ the odd part of $\left(X, \mathscr{O}_{X}\right)$. A superspace $\left(X, \mathscr{O}_{X}\right)$ is called a complex superspace if the even part $\left(X, \mathscr{O}_{X, 0}\right)$ is a complex space (with nilpotent elements) and the odd part $\mathscr{O}_{X, 1}$ of the structure sheaf $\mathscr{O}_{X}$ is a coherent sheaf of $\mathscr{O}_{X, 0}$-modules. This definition is attributed to P. Deligne. Thus, a complex superspace $\left(X, \mathscr{O}_{X}\right)$ can be considered to consist of a usual complex space (with nilpotents) $\left(X, \mathscr{O}_{X, 0}\right)$, a coherent sheaf $\mathscr{O}_{X, 1}$ of $\mathscr{O}_{X, 0^{-}}$ modules, and an anticommuting multiplication rule $\mu: \mathscr{O}_{X, 1} \otimes_{\mathscr{O}_{X}} \mathscr{O}_{X, 1} \rightarrow \mathscr{O}_{X, 0}$ giving to $\mathscr{O}_{X, 0} \oplus \mathscr{O}_{X, 1}$ the structure of a sheaf of supercommutative rings. Hence it has more structure than a graded complex space. In addition to the presence of nilpotent elements, one also encounters commuting and anticommuting variables in the study of complex supergeometry.

Morphisms over $\mathbb{C}$ of complex superspaces are defined as usual taking care that the $\mathbb{Z}_{2}$-gradation is respected. The category of germs of complex superspaces is easily seen to be dual to the category of analytic superalgebras. A complex supermanifold is a complex superspace $\left(X, \mathscr{O}_{X}\right)$ such that $\left(X, \mathscr{O}_{X} / \mathscr{N}\right)$ is a complex manifold, $\mathscr{N} / \mathscr{N}^{2}$ is locally free over $\mathscr{O}_{X} / \mathscr{N}$, and $\mathscr{O}_{X}$ is locally isomorphic to the $\mathbb{Z}_{2}$-graded exterior algebra $\Lambda_{X}\left(\mathscr{N} / \mathscr{N}^{2}\right)$. From the point of view of deformations and moduli, it is more natural to consider compact complex superspaces than to consider compact complex supermanifolds, especially because Kuranishi's method [Ku] of constructing a versal family for a compact complex manifold depends on harmonic theory, and as yet there does not exist a superharmonic theory. We now state two basic definitions in the deformation theory of complex superspaces.

Let $(S, 0)$ be the germ of a complex superspace. Let $\left(X, \mathscr{O}_{X}\right)$ be a given compact complex superspace. A family of deformations of $\left(X, \mathscr{O}_{X}\right)$ parametrized by the germ $(S, 0)$ is a proper flat holomorphic map $\pi: \mathscr{X} \rightarrow S$, together with an imbedding $i: X \hookrightarrow \mathscr{X}$, identifying $X$ with the fiber $\pi^{-1}(0)$. Morphisms of families of deformations of $\left(X, \mathscr{O}_{X}\right)$ parametrized by $(S, 0)$ are defined as usual. Let $(\mathscr{X}, \pi, S, 0)$ be a family of deformations of $\left(X, \mathscr{O}_{X}\right)$. Let $\left(S^{\prime}, 0^{\prime}\right)$ be any complex superspace germ and let $f:\left(S^{\prime}, 0^{\prime}\right) \rightarrow(S, 0)$ be a morphism. By base change $f$ induces a family of deformations of $\left(X, \mathscr{O}_{X}\right)$, denoted by $\mathscr{X}_{f}$, parametrized by $\left(S^{\prime}, 0^{\prime}\right)$. This family $\mathscr{X}_{f}$ is said to be induced by $f$.

A family of deformations $(\mathscr{Z}, \pi, S, 0)$ of $\left(X, \mathscr{O}_{X}\right)$ is said to be semiuniversal (or miniversal), if every other deformation $\left(\mathscr{Y}, \sigma, S^{\prime}, 0^{\prime}\right)$ of $\left(X, \mathscr{O}_{X}\right)$ is obtained from $\mathscr{X}$ by base change $\left(S^{\prime}, 0^{\prime}\right) \stackrel{f}{\rightarrow}(S, 0)$, and the differential $d f$ of $f$ is unique. A semiuniversal family $(\mathscr{X}, \pi, S, 0)$ of deformations of $\left(X, \mathscr{O}_{X}\right)$ is said to be universal if the inducing map $f$ is unique. 
We could have defined the notions of deformations and versal families by taking the parameter spaces to be the usual complex spaces (the purely even complex superspaces). However, this restriction would be rather unwarranted since it is known that moduli of compact complex supermanifolds depend on odd parameters. Hence it is necessary to be quite general; we should work, as we do in this paper, in the category of complex superspaces over complex superspaces with fibers being compact complex superspaces.

Similar to the above definitions of deformations and versal deformations, we could formulate notions of deformations and versal deformations for other complex supergeometric objects like superholomorphic vector bundles, coherent sheaves, cohomology classes, etc. We do not state explicitly these definitions. For brevity, in the rest of this paper by an analytic s-algebra (resp. complex s-space) we mean an analytic superalgebra (resp. complex superspace).

In order to prove the existence of a versal family of deformations for a compact complex s-space and to prove other existence theorems, we work, as stated earlier, in an abstract setting. We consider that the category of cofibered groupoids over the category of analytic s-algebras over a fixed analytic s-algebra is the appropriate set up, guided by the success of similar approaches in algebraic and analytic geometry (Rim [Ri], Artin $\left[\mathrm{A}_{2}\right]$, Bingener $\left.\left[\mathrm{Bi}_{2}\right]\right)$. To keep this introduction short, we do not state the basic definitions and 'construction techniques' of this general abstract setting, referring the reader to the relevant sections $1.12,6,7$, and 8 of this paper .

This paper is organized as follows. Analytic s-algebras are studied in $\S 1$. The principal results are the superanalogues of Artin's approximation theorems (Theorems (1.11) and (1.13)). The main result (Corollary (2.8)) of $\S 2$ is that a dualizing complex exists for any compact complex s-space. In $\S 3$, following $[\mathrm{Pa}$, $F_{1}$ ], we construct in the supergeometric sense cotangent and relative cotangent complexes. Deformations and extensions of complex s-spaces are studied in $\S 4$. The main result is Theorem (4.6) which gives the existence of a canonical isomorphism from the set $\operatorname{Ex}(X / S, \mathscr{M})$ of equivalence classes of extensions of $X / S$ by a module $\mathscr{M}$ to the set $T_{X / S}^{1}(\mathscr{M})_{\text {ev }}$ of even elements of $T_{X / S}^{1}(\mathscr{M})$. The obstructions to extending deformations are described. It is shown (Theorem (4.8)) that there is a canonical element $\operatorname{ob}\left[S^{\prime}\right] \in T_{X / S}^{2}\left(f^{*} \mathscr{N}\right)$ (where $\mathscr{N}$ is the coherent module giving the extension $S^{\prime}$ of $S$ ) which vanishes if and only if the deformation $X / S$ can be extended to $S^{\prime}$. In $\S 5$, we generalize the method of $\left[\mathrm{F}_{2}\right]$ of calculating relative Ext-sheaves in the category of complex s-spaces. $\S 6$ gives an account of the formal deformation theory of compact complex sspaces. In particular, (6.6) describes Schlessinger's sufficient conditions for the existence of a formally versal formal family and Schlessinger's theorem is proved in (6.14). Theorem (6.16) gives a criterion for openness for the property of being formally versal. It is shown $((6.19),(6.20))$ that formal versality is an open condition for deformations of compact complex s-spaces, for deformations of coherent sheaves, and for deformations of quotients.

Several representability criteria and representability theorems are proved in §7. In particular, Proposition (7.3) (superanalogue of Schuster's theorem) gives a sufficient condition under which a formal versal family is versal, Theorem (7.5) gives sufficient conditions under which a functor $F$ from the category of complex s-spaces over a fixed complex s-space to the category of sets can be rep- 
resented by a complex s-space (not necessarily separated) and Proposition (7.8) shows that $F$ has a coarse moduli space if its sheafification is representable.

Several important but technical results are proved in $\S 8$. In $(8.2)$ we give sufficient conditions which will imply the crucial (L3)-condition in the representability criteria of $\S 7$. These conditions are then verified for compact s-spaces and modules with compact support. The essential point here is that a compact complex s-space as well as a module with compact support admits an even versal deformation, see (8.4) and (8.9).

In the final section, $\S 9$, we give five important applications of our results in the earlier sections. (a) The existence of a versal family of deformations parametrized by a complex s-space for any coherent sheaf with compact support over a complex s-space is proved (Theorem (9.1)); (b) it is shown that the Hilbert functor (in the category of complex s-spaces) is representable (Theorem (9.2)); (c) the existence of a versal family of deformations (parametrized by a complex s-space) for any compact complex s-spaces is proved (Theorem (9.5)); (d) the existence of a coarse moduli space for coherent simple sheaves is proved (Theorem (9.8)); and (e) it is shown that the Picard functor is representable by a complex s-space (not necessarily separated).

Work on this paper started in Summer 1986 when the second author visited Göttingen. In the meantime Weintrob has also given a proof of (9.1) and (9.5) in $\left[\mathrm{W}_{2}\right]$ in the case of complex supermanifolds. His proof is different from ours. However, in both papers Banach analytic methods are avoided and both use Artin's approximation theorem for deriving the existence of versal deformation from the existence of even versal deformation, which is proved by using the solutions of the moduli problems in the classical complex analysis.

We thank J. Bingener, O. Forster, and S. Kosarew for several discussions relating to this work and P. Deligne for his letter [De] explaining his viewpoint on duality theory in supergeometry. The second author would like to thank J. Rabin for explaining to him the physicists' view(s) of supergeometry; he expresses his thanks to CINVESTAV (Mexico City), SFB (Göttingen), UCLA (Los Angeles), and UCSD (La Jolla) for their support.

Notations. In the following, the word super will be abbreviated by s, e.g., we will speak about s-spaces or s-algebras instead of superspaces or superalgebras. By $\mathbb{C}^{a \mid b}$ we denote the complex number space in $a$ even and $b$ odd dimensions (see, e.g., $\left[\mathrm{Ma}_{2}\right]$ ). If $\wedge$ is an analytic s-algebra (see $\S 1$ ) then $s \mathscr{C}_{\wedge}$ (resp. $\mathscr{C}_{\wedge}, \quad$ Alg $/ \wedge$ ) will be the category of all analytic $\wedge$-s-algebras (resp. analytic (even) $\wedge$-algebras, resp. all (even) $\wedge$-algebras). Similarly, s-An $/ S$ indicates the category of complex s-spaces over the fixed complex s-space $S$. The reduction of an s-space $T$ is denoted by $T_{\text {rd }}$, i.e., $\mathscr{O}_{T_{\mathrm{rd}}}=\mathscr{O}_{T} / \mathscr{F}$, where $\mathscr{F} \subset \mathscr{O}_{T}$ is the maximal nilpotent ideal sheaf of $\mathscr{O}_{T}$. Let $X \stackrel{f}{\rightarrow} S$ be a morphism of s-spaces. The category of modules (in the supersense, see $\S 1$ ) over $X$ will be denoted by $\operatorname{Coh}(X)$ whereas $\operatorname{Coh}_{p \mid f}(X)$ is the set of those coherent modules on $X$ which are proper and flat over $S$. For $\mathscr{O}_{X}$-modules $\mathscr{M}, \mathcal{N}$, one can form the relative $\mathscr{E} x t$-sheaves $\mathscr{E} x t_{f}^{i}(\mathscr{M}, \mathscr{N})$ which is defined to be the $i$ th cohomology of the complex $R f_{*} R \mathscr{H} \operatorname{om}_{X}(\mathscr{M}, \mathscr{N})$. For a point $s$ in $S$ and an $\mathscr{O}_{S}$-module $\mathscr{N}$, $\mathscr{N}(s)$ indicates the sheaf $\mathscr{N} / \mathfrak{m}_{s} \mathscr{N}$, where $\mathrm{m}_{s} \subset \mathscr{O}_{S}$ is the maximal ideal sheaf of the point $s$. Similarly, for a sheaf $\mathscr{M}$ on $X$ we call $\mathscr{M}(s):=\mathscr{M} / \mathfrak{m}_{s} \mathscr{M}$ the fiber of $\mathscr{M}$ over $s$. Finally, if $X$ is as above, we can associate to a coherent 
$\mathscr{O}_{X}$-module $\mathscr{M}$ the trivial extension of $X$ by $\mathscr{M}$ by which we mean the complex s-space $X[\mathscr{M}]=\left(X, \mathscr{O}_{X[\mathscr{K}]}\right)$ with $\mathscr{O}_{X[\mathscr{M}]}=\mathscr{O}_{X} \oplus \mathscr{M}$. Here the s-algebra structure is given by $\mathscr{M}^{2}=0$ and the $\mathbb{Z}_{2}$-grading by $\mathscr{O}_{X[\mathscr{K}], \varepsilon}=\mathscr{O}_{X, \varepsilon} \oplus \mathscr{M}_{\varepsilon}$.

\section{Analytic S-Algebras}

In the following, by an analytic s-algebra we mean a superalgebra $A=A_{0} \oplus$ $A_{1}$ such that $A_{0}$ is a (convergent) analytic $\mathbb{C}$-algebra in the usual sense (i.e., a quotient of a convergent power series ring over $\mathbb{C}$ ) and $A_{1}$ is finite as an $A_{0}$-module.

(1.1) We call $A$ a free analytic s-algebra over $\mathbb{C}$ if $A_{0} \cong \mathbb{C}\left\{X_{1}, \ldots, X_{n}\right\}$ and $A \cong \bigwedge M$ with $M:=A_{0}^{m}$. If $Y_{1}, \ldots, Y_{m}$ is a basis of the free $A_{0}$-module $M$ then we also write $\mathbb{C}\left\{X_{0}, \ldots, X_{m} ; Y_{1}, \ldots, Y_{m}\right\}$ for $A$. The $X_{i}$, resp. $Y_{j}$, are called the generators of even, resp. odd, degree.

The modules over an analytic s-algebra $A$ are always assumed to be $\mathbb{Z}_{2}$ graded, i.e., $M=M_{0} \oplus M_{1}$. All modules are assumed to be right modules. If $M$ is a right module then $M$ is equipped with a natural left structure by setting

$$
a m:=(-1)^{\operatorname{deg}(a) \operatorname{deg}(m)} m a,
$$

and obviously these structures are compatible.

(1.2) If $M$ is an $A$-module then by $M[1]$ we mean the module with $M[1]_{i}:=$ $M_{i+1}$, where for $m \in M[1], a \in A$ the product $m a$ is the given product in $M$, i.e., $M$ and $M[1]$ are isomorphic as abstract right modules. Obviously, $M[\alpha][\beta] \cong M[\alpha+\beta]$ for $\alpha, \beta \in \mathbb{Z}_{2}$ where we set $M[0]:=M$.

Throughout this paper, an odd, resp. even, morphism of $A$-modules $\phi: M \rightarrow$ $N$ is always a morphism of right modules with $\phi\left(M_{i}\right) \subseteq N_{i+1}$, resp. $\phi\left(M_{i}\right) \subseteq$ $N_{i}$. An even morphism is also a morphism of left modules whereas an odd morphism induces a morphism $M \rightarrow N$ [1] compatible with the right and left structure.

(1.3) If $M, N$ are $A$-modules then the set of all morphisms of right modules $\operatorname{Hom}_{A}(M, N)$ can be decomposed,

$$
\operatorname{Hom}_{A}(M, N) \cong \operatorname{Hom}_{A}(M, N)_{\mathrm{ev}} \otimes \operatorname{Hom}_{A}(M, N)_{\text {odd }},
$$

where the index ev, resp. odd, denotes the even, resp. odd, morphisms. Then $\operatorname{Hom}_{A}(M, N)$ carries a natural structure of an $A$-module by setting $(f \cdot a)(m)$ $:=f(a m)$ if $a \in A, f \in \operatorname{Hom}_{A}(M, N), m \in M$.

(1.4) If $M_{1}, \ldots, M_{n}$ are $A$-modules then one can form their tensor product $M_{1} \otimes_{A} \cdots \otimes_{A} M_{n}$ (see [L]). We remark that there is a natural isomorphism (of even degree)

given by

$$
\left(M_{1} \otimes \cdots \otimes M_{n}\right)[n] \stackrel{\sim}{\rightarrow} M_{1}[1] \otimes \cdots \otimes M_{n}[1]
$$

$$
m_{1} \otimes \cdots \otimes m_{n} \mapsto(-1)^{\mu} m_{1} \otimes \cdots \otimes m_{n},
$$

where $\mu=\sum_{j \geq 0} \operatorname{deg}\left(m_{n-1-2 j}\right)$. Two important quotients of the tensor product $M \otimes \cdots \otimes M^{\prime}(n$ times $)$ are the symmetric product $S^{n} M$ and the exterior product $\Lambda^{n} M$. Here $S^{n} M$ and $\Lambda^{n} M$ are the quotients with respect to the submodules generated by elements of the form

$$
m_{1} \otimes \cdots \otimes m_{n}-(-1)^{\mu_{i} \mu_{i-1}} m_{1} \otimes \cdots \otimes m_{i-2} \otimes m_{i} \otimes m_{i-1} \otimes m_{i+1} \otimes \cdots \otimes m_{n},
$$


resp.

$$
m_{1} \otimes \cdots \otimes m_{n}+(-1)^{\mu_{i} \mu_{i-1}} m_{1} \otimes \cdots \otimes m_{i-2} \otimes m_{i} \otimes m_{i-1} \otimes m_{i+1} \otimes \cdots \otimes m_{n},
$$

where $\mu_{i}:=\operatorname{deg}\left(m_{i}\right)$. The symmetric product of the elements $m_{1}, \ldots, m_{n} \in$ $M$ will be denoted by $m_{1} \vee \cdots \vee m_{n}$ whereas their exterior product will be denoted by $m_{1} \wedge \cdots \wedge m_{n}$. It is easy to see that with this definition, $S^{n}(M[1]) \cong$ $\left(\bigwedge^{n} M\right)[n]$, where the isomorphism is induced by the isomorphism

$$
(M \otimes \cdots \otimes M)[n] \cong M[1] \otimes \cdots \otimes M[1]
$$

described above. Clearly, the tensor product, symmetric product, and exterior product can be characterized by suitable universal properties which the reader is invited to formulate.

(1.5) If $A$ and $B$ are analytic s-algebras then one can form their analytic tensor product by setting

$$
A \tilde{\otimes}_{\mathbb{C}} B=A_{0} \tilde{\otimes}_{\mathbb{C}} B_{0} \oplus A_{1} \tilde{\otimes}_{\mathbb{C}} B_{1} \oplus A_{0} \tilde{\otimes}_{\mathbb{C}} B_{1} \oplus A_{1} \tilde{\otimes}_{\mathbb{C}} B_{0},
$$

where on the right-hand side $\tilde{\otimes}$ denotes the usual analytic $\otimes$-product. Then $A \tilde{\otimes}_{\mathbb{C}} B$ is again an analytic s-algebra in a straightforward way. Slightly more generally, it is possible to form a fibered analytic tensor product $A \tilde{\otimes}_{\Lambda} B$ if $\Lambda \rightarrow A, \Lambda \rightarrow B$ are morphisms of analytic s-algebras. Here $A \tilde{\otimes}_{\Lambda} B$ is the quotient of $A \tilde{\otimes}_{\mathbb{C}} B$ given by the ideal which is generated by the elelements $\lambda \otimes 1-1 \otimes \lambda, \lambda \in \Lambda$.

If $A \rightarrow B$ is a morphism of analytic s-algebras, then $B$ will be called a smooth $A$-s-algebra if $B$ is isomorphic to $A \tilde{\otimes}_{\mathbb{C}} B_{0}$, where $B_{0}$ is a free analytic s-algebra (see (1.1)), i.e., $B \cong A\left\{X_{1}, \ldots, X_{n} ; Y_{1}, \ldots, Y_{m}\right\}$, where the $X_{i}$ and $Y_{i}$ are generators of even, resp. odd, degree.

(1.6) By a derivation of degree $\alpha \in \mathbb{Z}_{2}$ we mean a $\mathbb{C}$-linear map $\delta: A \rightarrow M$ of degree $\alpha$ satisfying the product rule:

$$
\delta(a b)=\delta(a) \cdot b+(-1)^{\alpha \operatorname{deg}(a)} a \delta(b) .
$$

As in the classical case (see, e.g., [SS]), for every morphism of analytic s-algebras $A \rightarrow B$, there exists a module of 1 -forms $\Omega_{B / A}^{1}$ which is finite over $B$ and is characterized by the following universal property: There is given an $A$-linear derivation of degree $0, d: B \rightarrow \Omega_{B / A}^{1}$, and if $\delta: B \rightarrow M$ is any other $A$-linear derivation of degree $\alpha$ into some finite $B$-module $M$ then there is a unique factorization $\delta=h d$ with a homomorphism $h: \Omega_{B / A}^{1} \rightarrow M$ of $B$-modules of degree $\alpha$.

$\Omega_{B / A}^{1}$ and $d$ can be constructed similarly as in the classical case: If $B$ is a free analytic $A$-s-algebra with generators $X_{1}, \ldots, X_{n}$ of even degree and $Y_{1}, \ldots, Y_{m}$ of odd degree then $\Omega_{B / A}^{1}$ is the free $B$-module on the generators $d X_{1}, \ldots, d X_{n}, d Y_{1}, \ldots, d Y_{m}$, where $\operatorname{deg}\left(d\left(X_{i}\right)\right)=0, \operatorname{deg}\left(d Y_{j}\right)=1$. If $C$ is a quotient $B / \mathfrak{a}$ with $B$ as above then

$$
\Omega_{C / A}^{1} \cong \Omega_{B / A}^{1} /\left(\mathfrak{a} \Omega_{B / A}^{1}+B \cdot d \mathfrak{a}\right) \text {. }
$$

(1.7) Similarly as in the case of analytic algebras, in the supercase there is the notion of flatness. $A$ module $M$ over the analytic s-algebra $A$ is called flat if 
the functor $N \mapsto N \otimes_{A} M$ is exact on the category of all $A$-modules. Since $\otimes$ is compatible with direct limits it is of course sufficient to require the exactness for finite modules $N$. By using the Nakayama Lemma it is easy to see that a finite $A$-module is flat iff it is free. Moreover, an $A$-module $M$ is flat iff $M / \mathfrak{m}_{A}^{n} M$ is a flat $A / \mathfrak{m}_{A}^{n}$-module for every $n$. By using this latter property it is easy to see that an analytic tensor product of analytic s-algebras $A \tilde{\otimes}_{\mathbb{C}} B$ is flat over $A$.

(1.8) Proposition. Let $A \rightarrow B$ be a morphism of analytic s-algebras. Then $B$ is a smooth $A$-algebra iff $B$ is flat over $A$ and $\Omega_{B / A}^{1}$ is free.

Proof. There are homogeneous elements of degree 0 , say $x_{1}, \ldots, x_{n}$, and elements of degree 1 , say $y_{1}, \ldots, y_{m}$, in $\mathfrak{m}_{B}$ whose differentials generate $\Omega_{B / A}^{1}$ freely. Set $B^{\prime}:=A\left\{X_{1}, \ldots, X_{n} ; Y_{1}, \ldots, Y_{m}\right\}$ and let $B^{\prime} \rightarrow B$ be the $A$ homomorphism which is given by sending the $X_{i}$ to $x_{i}$ and $Y_{j}$ to $y_{j}$. Since $B^{\prime}$ and $B$ are flat over $A$ it is sufficient to prove that $B^{\prime} / \mathfrak{m}_{A} B^{\prime} \rightarrow B / \mathfrak{m}_{A} B$ is bijective, i.e., we may assume that $A=\mathbb{C}$ (see (1.9)(1)). From (1.9)(2) it follows that $B^{\prime} \rightarrow B$ must be surjective, i.e., $B \cong B^{\prime} / \mathfrak{a}$. Finally, (1.9)(3) shows that $\mathfrak{a}=0$.

In the proof above we have used

(1.9) Lemma. (1) (Preparation theorem) If $A \stackrel{\varphi}{\rightarrow} B$ is a quasifinite morphism of analytic s-algebras, i.e., $B / \mathfrak{m}_{A} B$ is a finite-dimensional vector space, then $A \stackrel{\varphi}{\rightarrow} B$ is finite.

(2) If $A$ is an analytic s-algebra then the map $\mathfrak{m}_{A} / \mathfrak{m}_{A}^{2} \rightarrow \Omega_{A / \mathbb{C}}^{1} / \mathfrak{m}_{A} \Omega_{A / \mathbb{C}}^{1}$ induced by $d$ is bijective.

(3) If $A=\mathbb{C}\left\{X_{1}, \ldots, X_{n} ; Y_{1}, \ldots, Y_{m}\right\}$ and $f \in \mathfrak{m}_{A}$ then $f=0$ iff $d f=0$ in $\Omega_{A / \mathbb{C}}^{1}$.

Proof. (1) is an immediate consequence of Serre's version of the preparation theorem since $A \rightarrow B$ is finite iff $A_{0} \rightarrow B_{0}$ is finite. (3) is an easy exercise in sderivations. For the proof of (2), consider the commutative algebra $\bar{A}:=A / \mathrm{m}_{A}^{2}$. Then $\Omega_{A / \mathbb{C}}^{1} / \mathfrak{m}_{A} \Omega_{A / \mathbb{C}}^{1} \cong \Omega_{A / \mathbb{C}}^{1} / \mathfrak{m}_{A} \Omega \frac{1}{A / \mathbb{C}}$ since $d\left(\mathfrak{m}_{A}^{2}\right) \subseteq \mathfrak{m}_{A} \Omega_{A / \mathbb{C}}^{1}$. Moreover $\mathfrak{m}_{\bar{A}}=$ $\mathfrak{m}_{A} / \mathfrak{m}_{A}^{2}$. Since (2) is well known for a commutative algebra, this concludes the proof.

Remark. Clearly the theory so far described also applies to Noetherian complete $\mathbb{C}$-s-algebras, i.e., algebras which are quotients of $\mathbb{C} \llbracket X_{1}, \ldots, X_{n} ; Y_{1}, \ldots, Y_{m} \rrbracket$, where the $X_{i}$, resp. $Y_{i}$, are generators of even, resp odd, degree (see (1.1) for the analytic case). In the sequel, we will use this without any further comment. For use in later sections, we prove the following.

(1.10) Lemma. Let $A \rightarrow B$ be a morphism of analytic s-algebras and $I \subseteq A$ an ideal with $I^{2}=0$. Assume that $A / I \rightarrow B / I B$ is flat and $I \otimes_{A} B \rightarrow I B$ is an isomorphism. Then $B$ is a flat A-algebra.

Proof. We must show that $\operatorname{Tor}_{1}^{A}(N, B)=0$ for every finite $A$-module $N$. Choose an exact sequence

$$
0 \rightarrow K \rightarrow A^{n} \rightarrow N \rightarrow 0
$$

and set $K^{\prime}:=K \cap I \cdot A^{n}$. After tensoring the diagram 


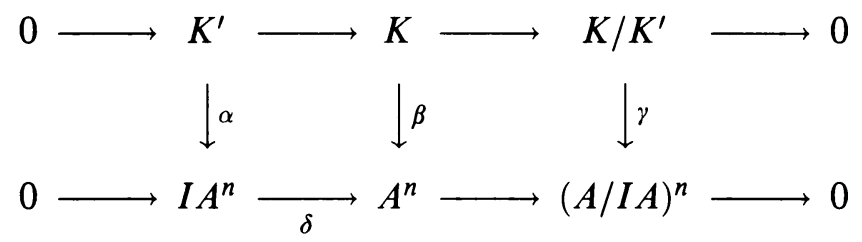

with $B$ we get the diagram

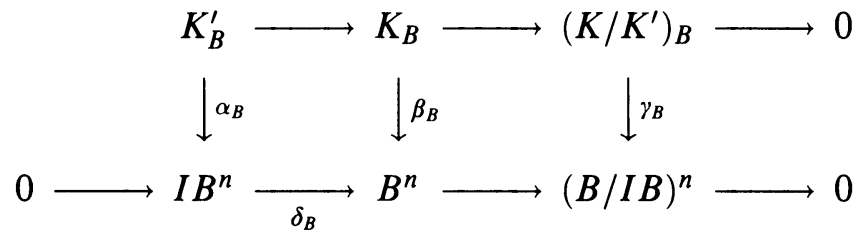

with exact lines. Observe that by our hypothesis, $\alpha_{B}, \gamma_{B}$, and $\delta_{B}$ are injective. Now an easy diagram chase shows $\operatorname{Tor}_{1}^{A}(N, B)=0$.

Next we will generalize the Artin approximation theorem to the case of analytic s-algebras. An analytic s-algebra $A$ is a local ring, and by $\widehat{A}$ we denote always its $\mathfrak{m}_{A}$-adic completion.

(1.11) Approximation Theorem I. Let $A \stackrel{j}{\rightarrow} B$ be a morphism of analytic salgebras and suppose that $\bar{\varphi}: B \rightarrow \widehat{A}$ is a formal section of $j$. Then for every $\mu \in \mathbb{N}$ there is a section $\varphi: B \rightarrow A$ of $j$ such that $\bar{\varphi} \equiv \varphi \bmod \mathfrak{m}_{A}^{n}$.

Proof. For an $A_{0}$-algebra $C$ (in the usual sense) we consider the set $F(C)$ of all isomorphism classes of pairs $\left(C^{*}, \varphi\right)$, where (1) $C^{*}$ is a noetherian $A$-s-algebra with $C_{0}^{*}=C$; (2) $\varphi: B \rightarrow C^{*}$ is an $A$-morphism; and (3) $C^{*}$ is finite as a $C$-module. Obviously, $F$ is in a natural way a functor $F: \operatorname{Alg} / A_{0} \rightarrow$ sets. The formal section $\bar{\varphi}$ gives rise to an element $(\widehat{A}, \bar{\varphi}) \in F\left(\widehat{A}_{0}\right)$. Hence our assertion follows from the usual approximation theorem (see $\left[\mathrm{A}_{1}, \mathrm{~A}_{2}\right]$ ) as soon as we can show that $F$ is a limit preserving functor. ${ }^{1}$ For this assume that $C=\lim C_{i}$ is a direct limit of analytic $A_{0}$-algebras. The $C$-module $C_{1}^{*}$ admits a presentation

$$
C^{r} \rightarrow C^{s} \rightarrow C_{1}^{*} \rightarrow 0,
$$

and the s-algebra structure of $C^{*}$ is given by a map $C_{1}^{*} \times C_{1}^{*} \rightarrow C$ which satisfies certain relations expressing the fact that $C^{*}$ is an s-algebra. It is easy to check that all these data can be realized over some $C_{i}$. Moreover, the map $\varphi: B \rightarrow C^{*}$ is given by maps $\varphi_{0}: B_{0} \rightarrow C, \varphi_{1}: B_{1} \rightarrow C_{1}^{*}$ which are compatible with the multiplication in $B$ and $C^{*}$. Clearly, $\varphi_{0}$ as well $\varphi_{1}$ arise from some index $i$, and by enlarging $i$ it is also possible to get a map $\varphi_{i}: B \rightarrow C_{i}^{*}$ of s-algebras.

Remark. Let the situation be as in (1.11) and $\mathfrak{a} \subset A$ an ideal. Suppose that there is already given a map $B \stackrel{\psi}{\rightarrow} A / \mathfrak{a}$ of $A$-s-algebras such that the diagram

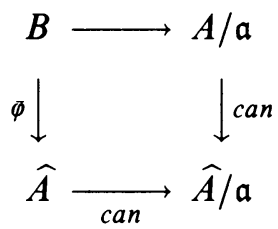

commutes. Then $\phi$ can be chosen in such a way that even $\phi=\psi \bmod a$.

\footnotetext{
${ }^{1}$ I.e., if $C=\lim _{\longrightarrow} C_{i}$ is a direct limit of analytic $A_{0}$-algebras then $F(C)=\underline{\lim } F\left(C_{i}\right)$.
} 
Proof. Let $A^{\prime}:=\bigwedge_{A}(A / \mathfrak{a}) \cong A \times A / \mathfrak{a}, B^{\prime}:=\bigwedge_{B}(A / \mathfrak{a})=B \times A / \mathfrak{a}$ (exterior algebra) where $A / \mathfrak{a}$ is regarded as a $B$-module via $\psi$. The morphism $\bar{\psi}$ induces an $A^{\prime}$-morphism $\bar{\phi}^{\prime}: B^{\prime} \rightarrow \widehat{A}^{\prime}$. By (1.11) we find a convergent section $\phi^{\prime}: B^{\prime} \rightarrow A^{\prime}$. After comparing with the canonical maps $B \rightarrow B^{\prime}$ and $A^{\prime} \rightarrow A$ we get a section $\phi$ of $j$ with the desired property.

For later purposes, it is convenient to generalize the approximation theorem in the spirit of $\left[\mathrm{A}_{2}\right]$. First we will introduce some notation.

(1.12) If $\Lambda$ is an analytic s-algebra let $s \mathscr{C}_{\Lambda}$ be the category of analytic $\Lambda$-salgebras and $s \hat{\mathscr{C}}_{\Lambda}$ the category of complete noetherian $\Lambda$-s-algebras with residue field $\mathbb{C}$. Suppose that $F: s \mathscr{C}_{\Lambda} \rightarrow$ sets is a functor on $s \mathscr{C}_{\Lambda}$ and consider

$$
\widehat{F}: s \hat{\mathscr{C}}_{\Lambda} \rightarrow \text { sets, } \quad \widehat{F}(\bar{A}):=\lim F\left(\bar{A} / \mathfrak{m}_{\bar{A}}^{n+1}\right) .
$$

Obviously, $\widehat{F}$ is a functor and for $A \in s \mathscr{C}_{\Lambda}$ there is a canonical map $F(A) \rightarrow$ $\widehat{F}(\widehat{A})$ denoted by $a \mapsto \hat{a}$. We will say that $F$ satisfies (L1) ((L2), (L3), resp.) if for every $\bar{A} \in s \hat{\mathscr{C}}_{\Lambda}$ and every representation $\bar{A}=\lim _{\longrightarrow} A^{(i)}$ as an inductive limit of analytic $\Lambda$-s-algebras $A^{(i)}$, the canonical map

$$
\lim _{\longrightarrow} F\left(A^{(i)}\right) \rightarrow \widehat{F}(\bar{A})
$$

has dense image (is injective, is surjective, resp.) (see $\left[\mathrm{Bi}_{1}\right]$ for the corresponding notions in the classical case). Observe that our condition (L3) is different from that in $\left[\mathrm{Bi}_{1}\right]$.

With these notations we will show

(1.13) Approximation Theorem II. Let $F$ be as above and assume that $F$ satisfies (L1). Then for every $n \in \mathbb{N}, B \in s \mathscr{C}_{\Lambda}$, and $\bar{b} \in \widehat{F}(\widehat{B})$ there is a $b \in F(B)$ such that $b \equiv \bar{b}$ modulo $\mathfrak{m}_{B}^{n}$.

Proof. Clearly, $\widehat{B}$ can be exhausted by analytic s-algebras over $B$, i.e., $\widehat{B}=$ $\lim _{i} B^{(i)}$. Because of $(\mathrm{L} 1)$ there is an index $i$ and $b_{i} \in F\left(B^{(i)}\right)$ whose image in $\widehat{F}(\widehat{B})$ coincides with $\bar{b}$ module $\mathfrak{m}_{\widehat{B}}^{n}$. By $(1.11)$, the formal section $B^{(i)} \stackrel{\Phi^{(i)}}{\rightarrow}$ $\widehat{B}$ can be approximated by a map $B^{(i)} \stackrel{\varphi^{(i)}}{\rightarrow} B$ with $\bar{\varphi}^{(i)} \equiv \varphi^{(i)}$ modulo $\mathfrak{m}_{B}^{n}$. Obviously, $b=\varphi_{*}^{(i)}\left(b_{i}\right)$ is the required approximation of $\bar{b}$.

\section{COMPLEX S-SPACES}

It is clear how to define ringed s-spaces. These are spaces $(X, \mathscr{R})$ where the sheaf of rings $\mathscr{R}$ on the topological space $X$ is equipped with a $\mathbb{Z}_{2}$-grading $\mathscr{R}=\mathscr{R}_{0} \oplus \mathscr{R}_{1}$ such that $\Gamma(U, \mathscr{R})$ is an s-algebra for every open set $U$ in $X$. The morphisms of ringed spaces are always assumed to be $\mathbb{Z}_{2}$-graded.

(2.1) A ringed s-space $\left(X, \mathscr{O}_{X}\right)$ over $\mathbb{C}$ is called a complex superspace if the underlying ringed space $X_{\text {ev }}:=\left(X, \mathscr{O}_{X, 0}\right)$ is a complex space with nilpotent elements and if $\mathscr{O}_{X, 1}$ is a coherent $\mathscr{O}_{X_{\mathrm{ev}}}$-module.

Observe that there is always a canonical map $X \stackrel{\pi}{\rightarrow} X_{\mathrm{ev}}$ which is finite. It is clear that the notion of coherent module makes sense on a complex sspace. We always assume as in $\S 1$ that the modules are $\mathbb{Z}_{2}$-graded. The morphisms are always assumed to be right morphisms. Obviously, one can form 
$\mathscr{H} \operatorname{om}(\mathscr{M}, \mathscr{N}), S^{n}(\mathscr{M}), \bigwedge^{n}(\mathscr{M})$, and $\mathscr{M}_{1} \otimes \cdots \otimes \mathscr{M}_{n}$ if $\mathscr{M}, \mathscr{N}$, and $\mathscr{M}_{i}$ are $\mathscr{O}_{X}$-modules. Clearly, these modules are coherent if this is the case for $\mathscr{M}, \mathscr{N}$, and the $\mathscr{M}_{i}$.

Moreover, the construction of the module of 1 -forms can be globalized. For a morphism of complex s-spaces $X \rightarrow Y$ it is denoted by $\Omega_{X / Y}^{1}$. A point $x$ of $X$ is called a smooth point if $\mathscr{O}_{X, x}$ is a free analytic s-algebra (see (1.1)). From (1.8) we obtain that the set of singular (i.e., nonsmooth) points of $X$ is the set $\operatorname{sing}\left(\Omega_{X}^{1}\right)$ of points $x \in X$ such that $\Omega_{X, x}^{1}$ is not a free $\mathscr{O}_{X, x}$-module. In particular, the set of smooth points of $X$ is open.

The category of all $\mathscr{O}_{X}$-modules $\left(\mathbb{Z}_{2}\right.$-graded as usual!) will be denoted by $\mathscr{M} o d(X)$. It contains enough injectives, as follows easily from the fact that for an injective $\mathscr{O}_{X_{\mathrm{ev}}}$-module $\mathscr{F}$ the module $\mathscr{H} o m_{\mathscr{O}_{X_{\mathrm{ev}}}}\left(\mathscr{O}_{X}, \mathscr{F}\right)$ is an injective $\mathscr{O}_{X}$-module. The derived category of $\mathscr{M}$ od $(X)$ is always denoted by $D(X)$. The symbols $D^{+}(X), D^{-}(X), D_{c}^{+}(X)$, etc. are used similarly as in the classical case (see, e.g., [Ha]).

If $f: X \rightarrow Y$ is a morphism of complex s-spaces and $\mathscr{M}$ is a $\mathscr{O}_{Y}$-module then we set $f^{*}(\mathscr{M}):=f^{-1}(\mathscr{M}) \otimes_{\mathscr{O}_{Y}} \mathscr{O}_{X}$, i.e., in forming the inverse image of a sheaf, the structure sheaf $\mathscr{O}_{X}$ is always tensored from the right. It is easily seen that as usual the functors $f_{*}$ and $f^{*}$ are adjoint functors. The following criterion for the coherence of a sheaf is very useful.

(2.2) Lemma. Let $X$ be a complex s-space and $X_{\mathrm{ev}}$ the underlying complex space. Then $\mathscr{M} \in \mathscr{M}$ od $(X)$ is coherent iff $\mathscr{M}$ is coherent as a module over $\mathscr{O}_{X_{\text {ev }}}$.

The simple proof is omitted. As an immediate corollary we get

(2.3) Corollary. (1) The structure sheaf of a complex s-space is a coherent sheaf of rings.

(2) If $f: X \rightarrow Y$ is proper then $f_{*}$ transforms coherent sheaves of $\mathscr{O}_{X}$-modules into coherent sheaves of $\mathscr{O}_{Y}$-modules.

(2.4) Remark. Similarly as above it is possible to define formal complex sspaces. By this we always mean a ringed s-space over $\mathbb{C}$ such that $X_{\mathrm{ev}}$ is a formal complex space and $\mathscr{O}_{X, 1}$ is a coherent module on $X_{\mathrm{ev}}$ (see $\left[\mathrm{Bi}_{2}\right]$ ). Clearly, (2.1)-(2.3) also hold for formal complex s-spaces.

(2.5) In the next sections we will need that a complex s-space $X$ admits a dualizing complex. For a complex $K_{X}^{\bullet} \in D_{c}^{b}(X)$ pointwise of finite injective dimension we consider the functor $D: D_{c}(X) \rightarrow D_{c}(X)$ given by

$$
D\left(\mathscr{M}^{\bullet}\right):=\operatorname{R\mathscr {H}}_{X}\left(\mathscr{M}^{\bullet}, K_{X}^{\cdot}\right) \text {. }
$$

There is always a natural biduality morphism

$$
\Phi\left(\mathscr{M}^{\bullet}\right): \mathscr{M}^{\bullet} \rightarrow D D\left(\mathscr{M}^{\bullet}\right)
$$

Then $K_{X}^{\circ}$ will be called a (pointwise) dualizing complex if the map above is an isomorphism for all $\mathscr{M}^{\cdot} \in D_{c}(X)$. As in [Ha, $\left.\mathrm{V}(8.1)\right]$, the dualizing complexes can be characterized as follows.

(2.6) Proposition. Let $K_{X}^{\cdot} \in D_{c}^{+}(X)$ have pointwise finite injective dimension. Then $K_{X}$ is a dualizing complex iff the canonical map $\Phi\left(\mathscr{O}_{X}\right): \mathscr{O}_{X} \rightarrow D D\left(\mathscr{O}_{X}\right)$ is an isomorphism. 
For the proof we remark that the same arguments as in [Ha] can be applied. We will now show that a dualizing complex on a complex s-space always exists. First we show

(2.7) Proposition. Let $f: X \rightarrow Y$ be a finite morphism of complex s-spaces and assume that $Y$ has a dualizing complex $K_{Y}^{*}$. Then

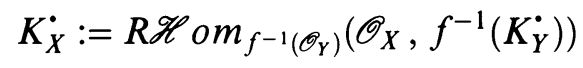

is a dualizing complex for $X$.

Proof. First we remark that $K_{X}^{\cdot}$ carries a natural $\mathscr{O}_{X}$-structure. By (2.6) it is sufficient to show that the map

$$
\mathscr{O}_{X} \rightarrow D_{X} D_{X}\left(\mathscr{O}_{X}\right)
$$

is bijective, where $D_{X}:=R \mathscr{H} o m_{X}\left(-, K_{X}^{\cdot}\right)$. But this follows from the fact that for $\mathscr{M}^{\cdot} \in D_{c}(X)$ there is a canonical isomorphism

$$
D_{X}\left(\mathscr{M}^{*}\right) \cong R \mathscr{H}^{o m_{f^{-1}\left(\mathscr{O}_{Y}\right)}}\left(\mathscr{M}^{\cdot}, K_{Y}^{\cdot}\right) .
$$

By applying this proposition to the morphism $X \rightarrow X_{\mathrm{ev}}$ and using [RR] we obtain

(2.8) Corollary. For every complex s-space there is a dualizing complex.

(2.9) Remarks. (1) In the next section we will also need that for every formal complex s-space there is at least locally a dualizing complex. This follows with the same arguments as above since $X_{\text {ev }}$ admits at least locally a dualizing complex since locally $X_{\mathrm{ev}}$ is embeddable into a smooth formal complex space.

(2) The full subcategory of $\mathscr{M} o d(X)$ consisting of all coherent $\mathscr{O}_{X}$-modules will always be denoted by $\operatorname{Coh}(X)$.

(3) P. Deligne, in his reply [De] to a question by the second author, stated that he has convinced himself that his approach (via Grothendieck's 'G-operations') explained in his appendix to Hartshorne's book [Ha] also works for complex superspaces (superschemes), yielding global duality theorems. In this letter Deligne also suggested that the usual (i.e., even) arguments should also carry over to give local duality theory.

\section{COTANGENT COMPLEX FOR COMPLEX S-SPACES}

We will first describe the theory for analytic s-algebras. We follow closely the exposition given in $\left[\mathrm{F}_{1}\right]$ (see also [Pa]).

(3.1) Let $A \rightarrow B$ be a fixed morphism of analytic s-algebras. Let $R$ be a $\left(\mathbb{Z}_{2} \times \mathbb{Z}\right)$-graded algebra over $A$. For a homogeneous element $x \in R$ of degree $(\alpha, \beta) \in \mathbb{Z}_{2} \times \mathbb{Z}$ denote by $\operatorname{deg}(x):=\alpha+\beta \bmod 2$ its total degree modulo 2 . By $R_{\alpha}^{\beta}$ we denote the subspace of homogeneous elements of type $(\alpha, \beta) \in \mathbb{Z}_{2} \times \mathbb{Z}$. Finally, $R^{\beta}$ will indicate the set $R_{0}^{\beta} \oplus R_{1}^{\beta}$. We will call $R$ an anticommutative $A$-s-algebra if the following three conditions are satisfied.

(1) $R$ is an s-algebra with respect to its total degree deg.

(2) The structure morphism $A \stackrel{\varphi}{\rightarrow} R$ is a morphism of s-algebras and $\varphi(A)$ $\subset R^{0}$.

(3) $R^{i}=0$ if $i>0, R^{0}$ is an analytic s-algebra, and $R^{i}$ is a finite $R^{0}$-module for all $i$. 
In the following, all the $R$-modules which occur are assumed to be $\left(\mathbb{Z}_{2} \times \mathbb{Z}\right)$ graded right modules. Now assume that there is given an $A$-linear derivation $\zeta$ on the anticommutative $A$-s-algebra $R$. We will call $(R, \zeta)$, or shortly $R$, a DG-s-algebra if $\zeta$ is homogeneous of degree $(0,1)$, i.e., $\zeta\left(R_{\alpha}^{\beta}\right) \subseteq R_{\alpha}^{\beta+1}$, and if $\zeta^{2}=0$.

By a morphism of DG-s-algebras $(R, \zeta) \rightarrow(S, \sigma)$ over $A$, we mean a morphism of $A$-algebras $R \rightarrow S$ which is bihomogeneous of degree $(0,0)$ and compatible with the differentials $\zeta, \sigma$.

Finally, it is possible to introduce DG-s-modules over a DG-s-algebra $R$ over $A$. Let $M$ be a $\left(\mathbb{Z}_{2} \times \mathbb{Z}\right)$-graded $R$-module equipped with a homogeneous map $\mu: M \rightarrow M$ of degree $(0,1)$ satisfying

$$
\mu(m r)=\mu(m) \cdot r+(-1)^{\operatorname{deg} m} m \zeta(r)
$$

for all homogeneous $m \in M, r \in R$. Then $(M, \mu)$, or simply $M$, is called a DG-s-module. Observe that $M$ is always considered as a right module over $R$, but according to the sign rule it then also has a natural left structure over $R$ !

In the following, an analytic s-algebra will often be regarded as a DG-algebra concentrated in the degrees $\mathbb{Z}_{2} \times 0$.

(3.2) We remark that any anticommutative s-algebra $R$ over $A$ can be regarded as an anticommutative algebra $\widetilde{R}$ by setting $\widetilde{R}^{\mu}=R_{0}^{\mu} \oplus R_{1}^{\mu+1}$. The same holds for modules, DG-s-algebras, etc. Hence a number of results from $\left[\mathrm{F}_{1}\right]$ can be quite directly generalized to the case of DG-s-algebras.

(3.3) For a morphism of anticommutative $A$-s-algebras $S \rightarrow R$ there is a

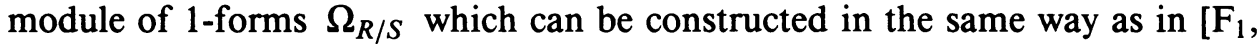
p. 4]. Then $\Omega_{R / S}$ is a $\left(\mathbb{Z}_{2} \times \mathbb{Z}\right)$-graded module over $R$ satisfying the usual universal property with respect to derivations. If $S \rightarrow R$ is a morphism of DG-s-algebras then $\Omega_{R / S}=\left(\Omega_{R / S}, \omega\right)$ carries the structure of a DG-s-module by setting for $x, y \in R$

$$
\omega(x d y):=\zeta(x) d y+(-1)^{\operatorname{deg} x} x d(\zeta(y)),
$$

where $d: R \rightarrow \Omega_{R / S}$ is the universal derivation on $R$.

(3.4) Similarly as in $\left[F_{1}\right]$ it is possible to introduce the notion of a free anticommutative s-algebra over $R$ (see [loc. cit. (1.1)(2)]: If $E$ is a set and $\operatorname{deg}: E \rightarrow \mathbb{Z}_{2} \times \mathbb{Z} \leq 0$ is a degree function, then by $R[E]$ we denote the free anticommutative $R$-s-algebra with generators $E$, i.e. $R[E] \cong \widetilde{S}\left(R^{E}\right)$, where $\widetilde{S}$ is the modified symmetric power as described in $\left[F_{1}\right.$, p. 3].

(3.5) We can now introduce the notion of a resolvent for a morphism of analytic s-algebras $A \rightarrow B$. Let $R$ be a DG-s-algebra over $A$ and $R \rightarrow B$ be an $A$ morphism. Then $R$ is said to be a resolvent for $B / A$ if (a) $R$ is a free $A$-algebra (see (3.4)) and (2) $R \rightarrow B$ is a quasi-isomorphism, i.e., $H^{0}(R) \cong B, H^{i}(R)=0$ if $i \neq 0$.

(3.6) Definition. Let $R$ be a resolvent for $B / A$. Then $L_{B / A}:=\Omega_{R / A} \otimes_{R} B$ is called the cotangent complex of $B / A$. For a $B$-module $M$, the functors

$$
T_{B / A}^{i}(M):=\operatorname{Ext}_{B}^{i}\left(L_{B / A}^{\cdot}, M\right), \quad T_{i}^{B / A}(M):=\operatorname{Tor}_{-i}^{B}\left(L_{B / A}^{\cdot}, M\right)
$$

are called the cotangent, resp. tangent, functors.

With exactly the same proofs as in $\left[F_{1}\right]$ one can easily show 
(3.7) Proposition. (1) $L_{B / A}^{\cdot}$ is well defined as an object of the derived category $D_{c}^{-}(B)$.

(2) An exact sequence $0 \rightarrow M^{\prime} \rightarrow M \rightarrow M^{\prime \prime} \rightarrow 0$ induces exact sequences

$$
\begin{aligned}
& \cdots \rightarrow T_{B / A}^{i}\left(M^{\prime}\right) \rightarrow T_{B / A}^{i}(M) \rightarrow T_{B / A}^{i}\left(M^{\prime \prime}\right) \rightarrow T_{B / A}^{i+1}\left(M^{\prime}\right) \rightarrow \cdots, \\
& \cdots \rightarrow T_{i}^{B / A}\left(M^{\prime}\right) \rightarrow T_{i}^{B / A}(M) \rightarrow T_{i}^{B / A}\left(M^{\prime \prime}\right) \rightarrow T_{i-1}^{B / A}\left(M^{\prime}\right) \rightarrow \cdots .
\end{aligned}
$$

(3) If $A \rightarrow B \rightarrow C$ are morphisms of analytic s-algebras then there is a triangle $L_{B / A}^{\cdot} \stackrel{\otimes}{=} \rightarrow L_{C / A}^{\cdot} \rightarrow L_{C / B}^{\cdot} \rightarrow L_{B / A}^{\cdot} \stackrel{\otimes}{=}[1]$ in the derived category.

(4) $T_{0}^{B / A}(M) \cong \Omega_{B / A}^{1} \otimes_{B} M, T_{B / A}^{0}(M) \cong \operatorname{Der}_{A}(B, M)$.

(5) If $A \rightarrow B$ is smooth then $T_{B / A}^{i}(M)=T_{i}^{B / A}(M)=0$ for $i>0$.

(6) Let $A \rightarrow A^{\prime}$ be another morphism of analytic s-algebras and set $B^{\prime}:=$ $B \otimes_{A} A^{\prime}$.

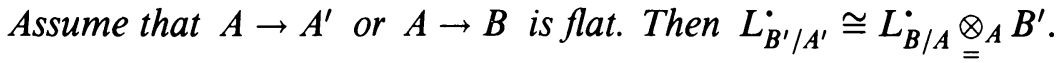

For the proofs, see $\left[F_{1}\right.$, pp. 11-15].

We will now define the cotangent complex $L_{X / Y}$ for morphisms of complex s-spaces $X \stackrel{f}{\rightarrow} Y$. For this we must have the notion of resolvent. We proceed as in $\left[F_{1}, \S 2\right]$.

(3.8) Recall that a simplicial scheme consists of a set $A_{0}$ and a system $A$ of finite subsets of $A_{0}$ called the simplices such that $A$ contains all subsets $\{a\}, a \in A_{0}$, and all subsets $\beta$ of $\alpha$ if $\alpha \in A$. A simplicial scheme of ringed s-spaces is a family $\left(W_{\alpha}\right)_{\alpha \in A}$ of ringed s-spaces $W_{\alpha}$ together with compatible morphisms $\rho_{\alpha \beta}: W_{\beta} \rightarrow W_{\alpha}$ if $\alpha \subseteq \beta \in A$. If the $W_{\alpha}$ are complex s-spaces (resp. Stein compact sets in some complex s-spaces $X_{\alpha}$, equipped with the sheaf $\left.\mathscr{O}_{X_{\alpha}} \mid W_{\alpha}\right)$ then we call $W_{*}:=\left(W_{\alpha}\right)_{\alpha \in A}$ a simplicial scheme of complex s-spaces (resp. s-Stein compact sets). For short, we call $W_{*}$ smooth if $\mathscr{O}_{W_{\alpha, x}}$ is a free analytic s-algebra for all $\alpha \in A, x \in W_{\alpha}$. Now a morphism of schemes of ringed s-spaces can be introduced as in [ $F_{1}$, p. 27]. Similarly, one can define $\mathscr{O}_{W_{*}}$-modules, coherence, and Ext-functors as described in [loc. cit].

(3.9) The most important example for these simplicial objects arises by a Stein covering of a complex space. Let $f: X \rightarrow Y$ be a morphism of complex sspaces and let $\left\{U_{i}\right\}_{i \in A_{0}}$ be a countable locally finite covering of $X$, where $U_{i}$ is Stein and open in $X$. Let $K_{i} \subseteq U_{i}$ be a Stein compact set and assume that (1) $\left\{\stackrel{\circ}{K}_{i}\right\}$ is a covering of $X$ and (2) there is a $Y$-embedding $U_{i} \hookrightarrow$ $W_{i} \times Y$ with some smooth space $W_{i}$. Set $A:=\left\{\alpha \subseteq I: K_{\alpha}:=\bigcap_{i \in \alpha} K_{i} \neq \varnothing\right\}$ and let $X_{*}=\left\{X_{\alpha}\right\}_{\alpha \in A}$ be the associated scheme of Stein compact sets with $\mathscr{O}_{X_{\alpha}}:=\mathscr{O}_{X} \mid K_{\alpha}$. Let $K_{\alpha} \stackrel{j_{\alpha}}{\rightarrow} \prod_{i \in \alpha} W_{i} \times Y=: Z_{\alpha}$ be the product of the embeddings $U_{i} \hookrightarrow W_{i} \times Y$ restricted to $K_{\alpha}$. Set $W_{\alpha}:=\left(K_{\alpha}, \mathscr{O}_{W_{\alpha}}\right)$ with $\mathscr{O}_{W_{\alpha}}:=j_{\alpha}^{-1}\left(\mathscr{O}_{Z_{\alpha}}\right)$. Then $W_{*}:=\left(W_{\alpha}\right)_{\alpha \in A}$ is a simplicial scheme of s-Stein compact sets which is smooth over $Y$. Moreover, $X_{*} \subseteq W_{*}$ is a closed subspace.

(3.10) Let $W_{*}=\left(W_{\alpha}\right)_{\alpha \in A}$ be an arbitrary simplicial scheme of s-Stein compact sets. We will introduce the notion of DG-algebra on $W_{*}$. By an $\mathscr{O}_{W_{*}}$-module we mean a collection of $\mathscr{O}_{W_{\alpha}}$-modules $\mathscr{M}_{\alpha}, \alpha \in A$, together with compatible morphisms of degree $0, \rho_{\alpha \beta}^{*}\left(\mathscr{M}_{\alpha}\right) \rightarrow \mathscr{M}_{\beta}$ if $\alpha \subseteq \beta \in A$. Similarly one can 
introduce the notion of a $W_{*}$-algebra $\mathscr{A}_{*}=\left(\mathscr{A}_{\alpha}\right)_{\alpha \in A}$. Such an algebra will be called an anticommutative s-algebra over $W_{*}$ if

(1) $\mathscr{A}_{*}=\bigoplus \mathscr{A}_{j *}^{i},(i, j) \in \mathbb{Z}_{2} \times \mathbb{Z}_{\leq 0}$, is $\left(\mathbb{Z}_{2} \times \mathbb{Z}\right)$-graded;

(2) the transition maps $e_{\alpha \beta}^{*}\left(\mathscr{A}_{\alpha}\right) \rightarrow \mathscr{A}_{\beta}$ are homogeneous degree 0 morphisms of algebras;

(3) $\mathscr{A}_{*}^{i}:=\mathscr{A}_{0 *}^{i} \oplus \mathscr{A}_{1 *}^{i}$ are coherent $\mathscr{O}_{W_{*}}$-modules; and

(4) $\mathscr{A}_{*}$ is anticommutative as explained in (3.1).

(3.11) Let $W_{*}$ be as in (3.10) and $\mathscr{A}_{*}$ an anticommutative s-algebra on $W_{*}$. It is clear how to define $\mathscr{A}_{*}$-modules and DG-s-modules over $\mathscr{A}_{*}$ if $\mathscr{A}_{*}$ is even a DG-s-algebra. For every $\alpha \in A$, there is a natural functor

$$
\rho_{\alpha}^{*}: \mathscr{M} \operatorname{od}\left(\mathscr{A}_{\alpha}\right) \rightarrow \mathscr{M} \operatorname{od}\left(\mathscr{A}_{*}\right)
$$

by setting $\rho_{\alpha}^{*}(\mathscr{N})_{\beta}:=\rho_{\alpha \beta}^{*}\left(\mathscr{M}_{\alpha}\right)$ if $\alpha \subseteq \beta$, and $\rho_{\alpha}^{*}(\mathscr{N})_{\beta}=0$ otherwise. Here $\mathscr{M} \operatorname{od}\left(\mathscr{A}_{\alpha}\right)$, resp. $\mathscr{M} \operatorname{od}\left(\mathscr{A}_{*}\right)$, denotes the category of modules over $\mathscr{A}_{\alpha}$, resp. $\mathscr{A}_{*}$. Then there is a natural isomorphism

$$
\mathscr{H} o m_{\mathscr{A}_{*}}\left(\rho_{\alpha}^{*}(\mathscr{N}), \mathscr{M}_{*}\right) \cong \mathscr{H} o m_{\mathscr{A}_{\alpha}}\left(\mathscr{N}, \mathscr{M}_{\alpha}\right) \text {. }
$$

An $\mathscr{A}_{*}$-module $\mathscr{M}_{*}$ is called free if it is isomorphic to a finite direct sum of modules of type $\rho_{\alpha}^{*}(\mathscr{N})$ with a free $\mathscr{A}_{\alpha}$-module $\mathscr{N}$ with generators of arbitrary bidegree in $\mathbb{Z}_{2} \times \mathbb{Z}_{<0}$.

Finally, let $\mathscr{A}_{*} \rightarrow \mathscr{B}_{*}$ be a morphism of anticommutative s-algebras on $W_{*}$. Then $\mathscr{B}_{*}$ is called a free algebra over $\mathscr{A}_{*}$ if $\mathscr{B}_{*}$ can be written as a union $\mathscr{B}_{*}=\bigcup_{i \geq 0} \mathscr{B}_{i *}$, where $\mathscr{B}_{0 *}=\mathscr{A}_{*}$, and $B_{i+1 *} \cong S_{B_{i *}}(\mathscr{F})$ is a symmetric algebra over some free $B_{i *}$-module $F$ in the sense above.

With these notations we are now able to introduce resolvents for complex s-spaces.

(3.12) Definition. Let $f: X \rightarrow Y$ be a morphism of complex s-spaces. A resolvent for $X / Y$ consists of

(a) a countable, locally finite open covering $\left\{U_{i}\right\}_{i \in I}$ of $X$ and closed embeddings $U_{i} \hookrightarrow W_{i} \times Y$ with $W_{i}$ smooth ; a covering $\left\{K_{i}\right\}_{i \in I}$ by compact Stein subsets with $\bigcup_{i \in I} \stackrel{\circ}{K_{i}}=X$ (let $X_{*} \hookrightarrow W_{*}$ be as in (3.9));

(b) a free DG-s-algebra $\mathscr{A}_{*}$ over $W_{*}$; and

(c) a morphism $\mathscr{A}_{*} \rightarrow \mathscr{O}_{X_{*}}$ which is a quasi-isomorphism of $\mathscr{O}_{W_{*}}$-algebras.

The data of a resolvent will be written shortly as $R=\left(X_{*}, W_{*}, \mathscr{A}_{*}\right)$.

(3.13) Let $X_{*}$ be as above. If $\mathscr{K}_{*}$ is a module over $X_{*}$ then we can associate to $\mathscr{M}_{*}$ a Čech-complex of sheaves denoted by $C^{*}\left(\mathscr{M}_{*}\right)$ (see $\left[\mathrm{F}_{1}\right.$, p. 32]). Then $j_{*}: \operatorname{Mod}\left(X_{*}\right) \rightarrow \operatorname{Mod}(X)$ is defined to be the functor $j_{*}\left(\mathscr{M}_{*}\right):=H^{0}\left(C^{\bullet}\left(\mathscr{M}_{*}\right)\right)$. The associated derived functor is denoted by $R j_{*}: D^{+}\left(X_{*}\right) \rightarrow D^{+}(X)$.

(3.14) Definition. If $R=\left(X_{*}, W_{*}, \mathscr{A}_{*}\right)$ is a resolvent for $X \rightarrow Y$ then

$$
L_{X / Y}^{\cdot}:=R j_{*}\left(\Omega_{\mathscr{A}_{*} / Y} \stackrel{\mathscr{A}_{*}}{\mathscr{O}_{X_{*}}}\right)
$$

is called the cotangent complex of $X / Y$.

(3.15) Theorem. (1) $L_{X / Y}^{\cdot}$ is well defined as an object of $D_{c}^{-}(X)$. 
(2) If

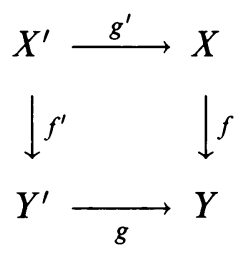

is a diagram of complex s-spaces then there is an induced morphism

$$
\operatorname{Lg}^{*}\left(L_{X / Y}^{\cdot}\right) \rightarrow L_{X^{\prime} / Y^{\prime}}^{\prime}
$$

For the proof we remark that the arguments of $\left[F_{1},(2.16)\right]$ can be easily carried over to the case of s-spaces. Now it is possible to introduce tangent and cotangent functors $T_{X / Y}^{i}(\mathscr{M})$ and $T_{i}^{X / Y}(\mathscr{M})$ as usual. There are similar exact sequences for the $T_{i}$ and $T^{i}$ as for analytic algebras. We leave the straightforward formulation to the reader.

Clearly, $L_{X / Y, x}$ is quasi-isomorphic to $L_{B / A}$ if $B:=\mathscr{O}_{X, x}, A:=\mathscr{O}_{Y, f(x)}$. Hence in the case of a smooth morphism $L_{X / Y}=\Omega_{X / Y}^{1}$ and so $T_{X / Y}^{i}(\mathscr{M})=$ $\operatorname{Ext}^{i}\left(\Omega_{X / Y}^{1}, \mathscr{M}\right) \cong H^{1}\left(\Theta_{X / Y} \otimes \mathscr{M}\right)$, where $\boldsymbol{\Theta}_{X / Y}:=\mathscr{H}_{o m}\left(\Omega_{X / Y}^{1}, \mathscr{O}_{X}\right)$ is the sheaf of vector fields of $X / Y$.

The following observation is used several times in this paper.

(3.16) Proposition. Let

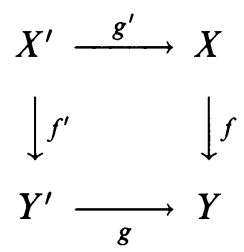

be a cartesian diagram of complex s-spaces and assume that $f$ or $g$ is flat. Then $L_{X^{\prime} / Y^{\prime}} \cong L g^{\prime *}\left(L_{X / Y}^{\cdot}\right)$.

Proof. It is sufficient to prove that the canonical map $L g^{\prime *}\left(L_{X / Y}^{\cdot}\right) \rightarrow L_{X^{\prime} / Y^{\prime}}$ is a quasi-isomorphism in each stalk. Hence it is sufficient to prove the analogous statement for analytic s-algebras, which follows from (3.7)(6).

(3.17) Let $f: X \rightarrow Y$ be a morphism of complex s-spaces and assume that $\mathscr{M}$ is a coherent $\mathscr{O}_{X}$-module. Then by $\mathscr{T}^{i}(X / Y ; \mathscr{M})$ we will denote the sheaf

$$
\mathscr{T}^{i}(X / Y ; \mathscr{M}):=\mathscr{E} x t_{f}^{i}\left(L_{X / Y}^{\cdot}, \mathscr{M}\right)=H^{i}\left(R f_{*} R \mathscr{H} \text { om }\left(L_{X / Y}^{\cdot}, \mathscr{M}\right)\right)
$$

on $Y$, whereas $\mathscr{T}_{X / Y}^{i}(\mathscr{M}):=\mathscr{E} x t_{X}^{i}\left(L_{X / Y}^{i}, \mathscr{M}\right)$.

\section{Deformations AND eXTensions OF COMPLEX S-SPACES}

(4.1) Let $X \rightarrow S$ be a morphism of complex s-spaces and assume that $\mathscr{M}$ is a coherent $\mathscr{O}_{X}$-module. As usual, by an extension of $X / S$ by $\mathscr{M}$ we mean a complex s-space $X^{\prime} \rightarrow S$ together with a fixed $S$-embedding $X \hookrightarrow X^{\prime}$ and an isomorphism $\mathscr{F} \cong \mathscr{M}$ of degree zero, where $\mathscr{F} \subseteq \mathscr{O}_{X^{\prime}}$ denotes the ideal of $X$. Two extensions $X^{\prime}, X^{\prime \prime}$ of $X / S$ by $\mathscr{M}$ are considered to be equivalent if there is an $S$-isomorphism $X^{\prime} \rightarrow X^{\prime \prime}$ compatible with the embeddings and the 
isomorphisms above. The set of equivalence classes of all extensions of $X / S$ by $\mathscr{M}$ will be denoted by $\operatorname{Ex}(X / S, \mathscr{M})$.

One main goal of this section is to prove that there is a canonical isomorphism of $\operatorname{Ex}(X / S, \mathscr{M})$ with the elements of $T_{X / S}^{1}(\mathscr{M})_{\mathrm{ev}}$. For the case of even complex spaces, this can be found in $\left[F_{1}\right]$ or at least partially in $[P]$. Since $\left[F_{1}\right]$ has not been published, it seems necessary for us to give at least an outline of the arguments in the s-case as far as we need it in the subsequent sections.

We will start with several lemmas.

(4.2) Let $\left(X_{*}, W_{*}, \mathscr{A}_{*}\right)$ be a resolvent for $X / S$ (see (3.12)). Let $X^{\prime}$ be an extension of $X / S$ by $\mathscr{M}$. Since $X=X^{\prime}$ as topological spaces, we can chop $X^{\prime}$ in the same way as $X$ and get an $X_{*}^{\prime}$. The $\mathscr{O}_{X_{*}}$-module associated to $\mathscr{M}$ will be denoted by $\mathscr{M}_{*}$. Then the following holds.

(4.3) Proposition. There is an S-morphism $\mathscr{A}_{*} \rightarrow \mathscr{O}_{X^{\prime}}$ making the diagram

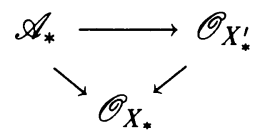

commutative. Any two such morphisms differ by a S-derivation $\delta: \mathscr{A}_{*} \rightarrow \mathscr{M}_{*}$.

Proof. By using (4.4) below we get an $S$-morphism $\mathscr{O}_{W_{*}} \rightarrow \mathscr{O}_{X^{\prime}}$ compatible with the projections onto $\mathscr{O}_{X_{*}}$. Now the arguments of $\left[\mathrm{F}_{1},(2.8)\right.$ and its proof $]$ show the existence of a map $\mathscr{A}_{*} \rightarrow \mathscr{O}_{X^{\prime}}$ as desired. That two such maps differ by an $S$-derivation is trivial.

(4.4) Lemma. There is an $S$-morphism $\mathscr{O}_{W_{*}} \rightarrow \mathscr{O}_{X !}$ compatible with the projections onto $\mathscr{O}_{X_{*}}$.

Proof. Let the notation be as in (3.9), (3.12). It is sufficient to show that for every $i \in I$ there is an $S$-map $\mathscr{O}_{W_{i}}\left|K_{i} \stackrel{\varphi_{i}}{\rightarrow} \mathscr{O}_{X^{\prime}}\right| K_{i}$ compatible with the projections onto $\mathscr{O}_{X} \mid K_{i}$. Clearly, since $W_{i}$ is a smooth space, this can be done locally. Hence there is a finite Stein covering $\mathscr{D}$ of $K_{i}$ such that there are $S$-maps $\mathscr{O}_{W_{i}}\left|V \stackrel{\varphi_{V}}{\longrightarrow} \mathscr{O}_{X^{\prime}}\right| V$ for $V \in \mathscr{D}$. On $V \cap V^{1}, V, V^{1} \in \mathscr{D}$, the differences $\varphi_{V}-$ $\varphi_{V^{1}}$ give rise to an element in the group $H^{1}\left(\mathscr{D}, \mathscr{D e r}\left(\mathscr{O}_{W_{i}}\left|K_{i}, \mathscr{M}\right| K_{i}\right)\right)$ which vanishes by Theorem B. By changing the $\varphi_{V}$ appropriately we may therefore assume that $\varphi_{V}-\varphi_{V^{1}}=0$ on $V \cap V^{1}$. This proves the existence of the lifting $\varphi_{i}$.

(4.5) Lemma. Let the notations be as in (4.2). Then the tangent functors $T_{X / S}^{k}(\mathscr{M})_{\varepsilon}, \varepsilon \in \mathbb{Z}_{2}$, are canonically isomorphic to the kth cohomology of the complex $\operatorname{Der}_{S}\left(\mathscr{A}_{*}, \mathscr{M}_{*}\right)_{\varepsilon} \cong \operatorname{Hom}_{X_{*}}\left(\Omega_{\mathscr{H}_{*} / S}, \mathscr{M}_{*}\right)_{\varepsilon}$. Moreover, assume that $X$ is $S$-flat and that $\mathscr{N}$ is a coherent $\mathscr{O}_{S}$-module. Then the canonical map

$$
\operatorname{Der}_{S}\left(\mathscr{A}_{*}, \mathscr{N} \otimes_{\mathscr{O}_{S}} \mathscr{A}_{*}\right) \rightarrow \operatorname{Der}_{S}\left(\mathscr{A}_{*}, \mathscr{N} \otimes_{\mathcal{O}_{S}} \mathscr{O}_{X}\right)
$$

is a quasi-isomorphism.

Here $\operatorname{Der}_{S}\left(\mathscr{A}_{*}, \mathscr{M}_{*}\right)_{\varepsilon}^{k}$ denotes the set all tuples of $S$-derivations $\mathscr{A}_{\alpha} \rightarrow \mathscr{M}_{\alpha}$ of degree $(\varepsilon, k) \in \mathbb{Z}_{Z} \times \mathbb{Z}$ compatible with the transition maps. For the proof we refer the reader to $\left[F_{1},(2.4),(3.7)(1)\right.$, and (3.10)], where the classical situation is considered. 
(4.6) Theorem. There is a canonical isomorphism

$$
\operatorname{Ex}(X / S ; \mathscr{M}) \rightarrow T_{X / S}^{1}(\mathscr{M})_{\mathrm{ev}}
$$

where $T_{X / S}^{1}(\mathscr{M})_{\mathrm{ev}}$ denotes the set of even elements of $T_{X / S}^{1}(\mathscr{M})$.

Proof. Let $R=\left(X_{*}, W_{*}, \mathscr{A}_{*}\right)$ be a resolvent of $X / S$ (see (3.12)). For $\left[X^{\prime}\right] \in$ $\operatorname{Ex}(X / S ; \mathscr{M})$ we choose $\mathscr{A}_{*} \rightarrow \mathscr{O}_{X^{\prime}}$ as in (4.3). Let $\zeta$ denote the differential of the DG-algebra $\mathscr{A}_{*}$. Then $\varphi \cdot \zeta: \mathscr{A}_{*} \rightarrow \mathscr{O}_{X^{\prime}}$ is an $S$-derivation of degree $(0,1) \in \mathbb{Z}_{2} \times \mathbb{Z}$ which gives rise to a homomorphism of $\mathscr{A}_{*}$-modules of degree $(0,1), \eta=\eta\left[X^{\prime}\right]: \Omega_{A_{*} / S} \rightarrow \mathscr{M}_{*}$, i.e., to an element of $T_{X / S}^{1}(\mathscr{M})_{\text {ev }}$ denoted by $\operatorname{cl}\left[X^{\prime}\right]$. By using the fact that any two morphisms $\varphi, \tilde{\varphi}: \mathscr{A}_{*} \rightarrow \mathscr{O}_{X_{*}}$ obtained as explained above only differ by an $S$-derivation, it is easy to see that $\operatorname{cl}\left[X^{\prime}\right]$ is well defined. On the other hand, let $[\eta] \in T_{X / S}^{1}(\mathscr{M})_{\mathrm{ev}}$ be given and assume that [ $\eta$ ] is represented by an $S$-derivation $\eta: \mathscr{A}_{*} \rightarrow \mathscr{M}_{*}$ of degree $(0,1)$. Then we can associate to $\eta$ an extension of $X / S$ by $\mathscr{M}$ in the following way: In the diagram

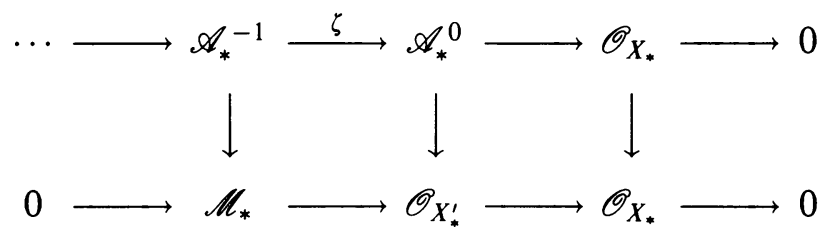

let $\mathscr{O}_{X^{\prime}}$ be the coherent $\mathscr{O}_{W_{*}}$-algebra $\mathscr{A}_{*}^{0} \amalg_{A^{-1}} \mathscr{M}_{*}$. By patching together we get from $\mathscr{O}_{X^{\prime}}$ an extension $X^{\prime}$ of $X / S$ by $\mathscr{M}^{*}$ in a natural way. It is easy to see that the maps $\left[X^{\prime}\right] \mapsto \operatorname{cl}\left[X^{\prime}\right]$ and $\eta \mapsto\left[X^{\prime}\right]$ constructed above are inverse to each other, and so (4.6) follows.

In the rest of this section we will show how to describe obstructions for extending deformations. We will give here an argument different from $\left[F_{1}\right]$, which does not depend on the heavy machinery of deformations of holomorphic mappings.

(4.7) Let $f: X \rightarrow S$ be a flat holomorphic mapping of complex s-spaces and $\mathscr{N}$ a coherent $\mathscr{O}_{S}$-module. Assume that $S^{\prime}$ is an extension of $S$ by $\mathscr{N}$. We will shortly say that the deformation $X \rightarrow S$ can be extended to $S^{\prime}$ if there is a commutative diagram

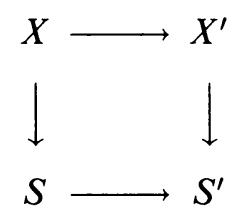

where $X^{\prime} \rightarrow S^{\prime}$ is flat. It is clear that $X^{\prime}$ then is an extension of $X$ by $f^{*}(\mathscr{N})$. (4.8) Theorem. There is a canonical element $\mathrm{ob}\left[S^{\prime}\right] \in T_{X / S}^{2}\left(f^{*} \mathscr{N}\right)_{\mathrm{ev}}$ which vanishes iff the deformation $X / S$ can be extended to $S^{\prime}$.

Proof. Let $\left(X_{*}, W_{*}, \mathscr{A}_{*}\right)$ be a resolvent for $X / S$. Using the notation introduced in (3.9) and (3.12), let $W_{*}^{\prime}$ be the simplicial scheme of s-Stein compact sets $W_{\alpha}^{\prime}:=\left(K_{\alpha}, \mathscr{O}_{Z_{\alpha}^{\prime}} \mid K_{\alpha}\right)$, where $Z_{\alpha}^{\prime}:=\prod_{i \in \alpha} W_{i} \times S^{\prime}$. Since $\mathscr{A}_{*}$ is a free $\mathscr{O}_{W_{*}-}$ algebra, it is possible to extend $\mathscr{A}_{*}$ to a free $\mathscr{O}_{W^{\prime}}$-algebra $\mathscr{A}_{*}^{\prime}$. Moreover, since a derivation on $\mathscr{A}_{*}$ is given by prescribing the images of free generators, it is also possible to extend the derivation $\zeta \in \operatorname{Der}_{S}\left(\mathscr{A}_{*}, \mathscr{A}_{*}\right)_{\mathrm{ev}}$ to an $S$-derivation 
$\zeta^{\prime} \in \operatorname{Der}_{S}\left(\mathscr{A}_{*}^{\prime}, \mathscr{A}_{*}^{\prime}\right)$, which again is of degree $(0,1)$. Then $\zeta^{\prime 2}: \mathscr{A}_{*}^{\prime} \rightarrow \mathscr{A}_{*}^{\prime}$ is an $S$-derivation of degree $(0,2)$. By construction, there is an exact sequence

$$
0 \rightarrow \mathscr{N} \otimes_{\mathscr{O}_{S}} \mathscr{A}_{*} \rightarrow \mathscr{A}_{*}^{\prime} \rightarrow \mathscr{A}_{*} \rightarrow 0
$$

and since $\zeta^{2}=0$, we obtain a map $\eta:=\zeta^{\prime 2}: \mathscr{A}_{*} \rightarrow \mathscr{N} \otimes_{\mathscr{O}_{S}} \mathscr{A}_{*}$ which is a cycle in the complex $\operatorname{Der}_{S}\left(\mathscr{A}_{*}, \mathscr{N} \otimes_{\mathscr{O}_{S}} \mathscr{A}_{*}\right)_{\mathrm{ev}}^{\bullet}$ of compatible $S$-derivations. This defines an element

$$
\mathrm{ob}\left[S^{\prime}\right]:=[\eta] \in T_{X / S}^{2}\left(f^{*} \mathscr{N}\right) \quad(\text { see }(4.5)) .
$$

It is easily verified that $o b\left[S^{\prime}\right]$ does not depend on the choices involved. Now assume that $X / S$ can be extended to a deformation $X^{\prime} / S^{\prime}$. Then we start with a resolvent $\left(X_{*}^{\prime}, W_{*}^{\prime}, \mathscr{A}_{*}^{\prime}\right)$ of $X^{\prime} / S^{\prime}$ and apply the construction above to $\mathscr{A}_{*}:=\mathscr{A}_{*}^{\prime} \otimes \mathscr{O}_{S^{\prime}} \mathscr{O}_{S}$, which because of the flatness of $X^{\prime} \rightarrow S^{\prime}$ is a resolvent for $X / S$. For $\zeta^{\prime}$ we can take the differential in the DG-s-algebra $\mathscr{A}_{*}^{\prime}$. Hence $\zeta^{\prime 2}=0$ and $\mathrm{ob}\left[S^{\prime}\right]=0$.

Assume conversely that the canonical element $\mathrm{ob}\left[S^{\prime}\right]=0$. Then $\zeta^{\prime 2}$ is a coboundary in $\operatorname{Der}_{S}\left(\mathscr{A}_{*}, \mathscr{N} \otimes \mathscr{A}_{*}\right)_{\text {ev }}$, i.e., there is an $S$-derivation $\mathscr{A}_{*} \stackrel{\delta}{\rightarrow}$ $\mathscr{N} \otimes \mathscr{A}_{*}$ of degree $(0,1)$ with $\zeta^{\prime 2}=\delta \zeta^{\prime}+\left(1 \otimes \zeta^{\prime}\right) \delta$. Replace $\zeta^{\prime}$ by $\zeta^{\prime \prime}:=\zeta^{\prime}-\delta$. Then $\zeta^{\prime \prime 2}=0$, i.e., $\left(A_{*}^{\prime}, \zeta^{\prime \prime}\right)$ is a DG-s-algebra. The sheaf $\mathscr{O}_{X^{\prime}}:=H^{0}\left(\mathscr{A}_{*}^{\prime}\right)$ can be patched together to give an $S^{\prime}$-space $X^{\prime}$. Since $(*)$ is exact, we get that $X^{\prime}$ is an extension of $X$ by $f^{*} \mathscr{N}$. Because of (1.10) $X^{\prime} \rightarrow S^{\prime}$ is flat. This completes the proof of (4.8).

(4.9) Remarks. (1) The construction of (4.6) is compatible with base change in the following sense. Let

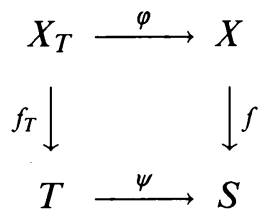

be a cartesian diagram of complex s-spaces and assume that $f$ is flat. Let $\mathcal{N}$ be a coherent $\mathscr{O}_{X}$-module. Then $L_{X_{T} / T}^{\bullet} \cong L \varphi^{*} L_{X / S}^{\bullet}$ by (3.16), and the canonical diagram

$$
\begin{gathered}
\operatorname{Ex}(X / S ; \mathscr{N}) \longrightarrow \operatorname{Ex}\left(X_{T} / T ; \varphi^{*} \mathscr{N}\right) \\
\mid \iota \\
T_{X / S}^{1}(\mathscr{N})_{\mathrm{ev}} \longrightarrow T_{X_{T} / T}^{1}\left(\varphi^{*} \mathscr{N}\right)_{\mathrm{ev}}
\end{gathered}
$$

commutes. This follows easily from the construction above applied to a resolvent $\left(X_{*}, W_{*}, \mathscr{A}_{*}\right)$ of $X / S$ and the resolvent $\left(\widetilde{X}_{*}, \widetilde{W}_{*}, \widetilde{\mathscr{A}_{*}}\right)$ of $X_{T} / T$ which we construct as follows: Let $\left\{V_{j}\right\}_{j \in J}$ be a countable locally finite, open Stein covering of $X_{T}$ such that $\varphi\left(V_{j}\right) \subseteq U_{\sigma(j)}$ for some $\sigma(j) \in I$, where $\left\{U_{i}\right\}$ is the covering associated to the given resolvent of $X / S$ (see (3.9), (3.12)). We can assume that there are Stein compact sets $L_{j}$ with $\bigcup \stackrel{\circ}{L}_{j}=X_{T}$ and $\varphi_{T}\left(L_{j}\right) \subseteq K_{i}$. From the given embedding $U_{\sigma(j)} \hookrightarrow W_{\sigma(j)} \times S$ we obtain by base change an embedding $V_{j} \hookrightarrow W_{\sigma(j)} \times T$. With the construction of (3.9) we obtain from 
these data the $\widetilde{X}_{*}, \widetilde{W}_{*}$. Finally, for $\widetilde{\mathscr{A}_{*}}$ we take the inverse image of $\mathscr{A}_{*}$ under the map $\widetilde{W}_{*} \rightarrow W_{*}$.

(2) A similar remark holds for the obstruction ob $\left[S^{\prime}\right]$. Let the notation be as in (1) and assume that

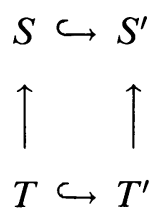

is a commutative diagram of s-spaces, where $S^{\prime}$, resp. $T^{\prime}$, is an extension of $S$ by $\mathscr{F}$, resp. $T$ by $\mathscr{J}$. Then under the canonical map $T_{X / S}^{2}\left(f^{*} \mathscr{F}\right) \rightarrow$ $T_{X_{T} / T}^{2}\left(f_{T}^{*} \mathscr{J}\right)$ the element ob $\left[S^{\prime}\right]$ is mapped onto $\mathrm{ob}\left[T^{\prime}\right]$ as is easily seen with the resolvent in (1).

(3) The results of this section also hold in the category of formal complex s-spaces. This will be used without any further comment in later sections.

\section{Relative EXT-SHEAVES FOR COMPLEX S-SPACES}

The purpose of this section is to generalize $\left[F_{2}\right]$ to the category of complex s-spaces and to give a few applications which will be important in later sections.

(5.1) Theorem. Let $f: X \rightarrow Y$ be a morphism of complex s-spaces and $\mathscr{G}$ a coherent $Y$-flat $\mathscr{O}_{X}$-module, whose support is proper over $Y$. Then there is a natural functor (depending on $\mathscr{G}$ )

$$
L f_{\#}: D_{c}^{-}(X) \rightarrow D_{c}^{-}(Y)
$$

with the following properties.

(1) For $\mathscr{F}^{\cdot} \in D_{c}^{-}(X), \mathscr{N}^{\cdot} \in D_{c}^{+}(Y)$ there is a natural isomorphism

$$
R f_{*} R \mathscr{H} o m_{X}\left(\mathscr{F}^{\cdot}, \mathscr{G} \otimes f^{*}\left(\mathscr{N}^{*}\right)\right) \cong R \mathscr{H} o m_{Y}\left(L f_{\#}\left(\mathscr{F}^{*}\right), \mathscr{N}^{*}\right) .
$$

(2) $f_{\#}$ is compatible with base change $Y^{\prime} \rightarrow Y$, i.e., if

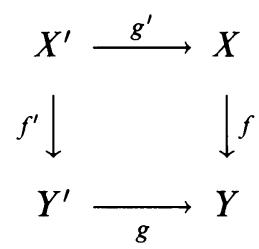

is cartesian then $L g^{*} L f_{\#} \cong L f_{\#}^{\prime} L g^{\prime *}$, where $L f_{\#}^{\prime}$ is the functor associated to $\mathscr{G}^{\prime}:=g^{\prime *}(\mathscr{G})$.

The proof in the case of even complex spaces $\left(\mathrm{see}\left[\mathrm{F}_{2}\right]\right)$, also applies to our more general situation, as the reader is invited to verify. Observe that there is a dualizing complex for $Y$ (see (2.8)), the existence of which is very essential for the proof in $\left[F_{2}\right]$

(5.2) Remark. By (2.9) there is at least locally also a dualizing complex for formal complex s-spaces. Therefore (5.1) also holds for morphisms of formal complex s-spaces $\bar{f}: \bar{X} \rightarrow \bar{Y}$, which is adic.

We will now give an application of (5.1) which will be important in $\S 8$. First we will fix the notation. 
(5.3) We suppose that $\bar{Y}=\left\{y_{0}\right\}$ is a formal complex s-space consisting of one point, and we denote by $\bar{A}$ the stalk $\mathscr{O}_{\bar{Y}, y_{0}}$ which is a complete local C-algebra. Assume that $\bar{A}=\underline{\lim }_{i} A^{(i)}$ is written as an inductive limit of (convergent) analytic s-algebras $\overrightarrow{A^{(i)}}$. Let $\left(Y^{(i)}, y_{0}\right)$ indicate the complex sspace germ associated to $A^{(i)}$ and denote by $p_{i}: \bar{Y} \rightarrow Y^{(i)}$ the canonical map. Assume now that $\bar{f}: \bar{X} \rightarrow \bar{Y}$ is a proper morphism obtained by base change $\bar{Y} \rightarrow Y^{\left(i_{0}\right)}$ from a proper morphism $X^{\left(i_{0}\right)} \stackrel{f_{i_{0}}}{\rightarrow} Y^{\left(i_{0}\right)}$. For $i \geq i_{0}$, set $X^{(i)}:=X^{\left(i_{0}\right)} \times \times_{Y^{\left(i_{0}\right)}} Y^{(i)}, f^{(i)}=f^{\left(i_{0}\right)} \times$ id. Let $\tilde{p}_{i}: \bar{X} \rightarrow X^{(i)}$ denote the morphism induced by $p_{i}$.

(5.4) Proposition. With the notation as in (5.3), assume that $\mathscr{G}_{i_{0}}$ is a $Y^{\left(i_{0}\right)}$-flat coherent module on $X^{\left(i_{0}\right)}, \mathscr{F}_{i_{0}} \in D_{c}^{-}\left(X^{\left(i_{0}\right)}\right)$, and $\mathscr{N}_{i_{0}} \in \operatorname{Coh}\left(Y^{\left(i_{0}\right)}\right)$. Set $\overline{\mathscr{G}}:=$ $\tilde{p}_{i_{0}}^{*}\left(\mathscr{G}_{i_{0}}\right), \overline{\mathscr{F}}^{\cdot}:=L \tilde{p}_{i_{0}}^{*}\left(\mathscr{F}_{i_{0}}^{\cdot}\right)$, and $\overline{\mathcal{N}}:=p_{i_{0}}^{*}\left(\mathscr{N}_{i_{0}}\right)$. Similarly, denote by $\mathscr{G}_{i}, \mathscr{F}_{i}^{\cdot}$, and $\mathscr{N}_{i}$ the induced objects on $X^{(i)}$ and $Y^{(i)}$ respectively if $i \geq i_{0}$. Then the canonical map of relative Ext-sheaves

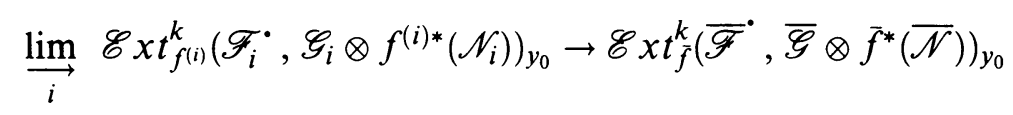

is bijective.

Proof. By (5.1)(1),

$$
\begin{aligned}
\mathscr{E} x t_{f^{(i)}}^{k}\left(\mathscr{F}_{i}^{\cdot}, \mathscr{G}_{i} \otimes f^{(i) *}\left(\mathscr{N}_{i}\right)\right) & \cong \mathscr{E} x t_{Y^{(i)}}^{k}\left(L f_{\#}^{(i)}\left(\mathscr{F}_{i} \cdot\right), \mathscr{N}_{i}\right), \\
\mathscr{E} x t_{f^{\prime}}^{k}\left(\overline{\mathscr{F}}^{\cdot}, \overline{\mathscr{G}} \otimes \bar{f}^{*}(\overline{\mathscr{N}})\right) & \cong \mathscr{E} x t_{\bar{Y}}^{k}\left(L \bar{f}_{\#}\left(\overline{\mathscr{F}}^{\cdot}\right), \overline{\mathcal{N}}\right) .
\end{aligned}
$$

Denote by $\mathscr{M}_{i}^{\cdot}$, resp. $\overline{\mathscr{M}}^{\cdot}$, the complex $L f_{\#}^{(i)}\left(\mathscr{F}_{i} \cdot\right)$, resp. $L \bar{f}_{\#}\left(\overline{\mathscr{F}}^{*}\right)$. By $(5.1)(2)$,

$$
\overline{\mathscr{M}}^{\cdot}=L p_{i}^{*}\left(\mathscr{M}_{i}^{*}\right), \quad \mathscr{M}_{i}^{*}=L p_{i i_{0}}^{*}\left(\mathscr{M}_{i_{0}}^{*}\right)
$$

where $p_{i j}: Y^{(i)} \rightarrow Y^{(j)}$ indicates the transition map. Now (5.4) follows from

(5.5) Lemma. Let $\mathscr{M}_{i_{0}}^{*} \in D_{c}^{-}\left(Y^{\left(i_{0}\right)}\right)$ be a complex on $Y^{\left(i_{0}\right)}$ and set $\mathscr{M}_{i}^{*}:=$ $L p_{i i_{0}}^{*}\left(\mathscr{M}_{i_{0}}^{*}\right)$ and $\overline{\mathscr{M}}^{\cdot}:=L p_{i}^{*}\left(\mathscr{M}_{i_{0}}^{*}\right)$ for $i \geq i_{0}$. Then for every coherent module $\mathscr{N}_{i_{0}}$ as in (5.4), the canonical map

$$
\mathrm{lim}_{\longrightarrow} \mathscr{E} x t_{Y^{(i)}}^{k}\left(\mathscr{M}_{i}^{\cdot}, \mathscr{N}_{i}\right)_{y_{0}} \rightarrow \mathscr{E} x t \frac{k}{\bar{Y}}\left(\overline{\mathscr{M}}^{\cdot}, \overline{\mathscr{N}}\right)_{y_{0}}
$$

is an isomorphism.

Proof. We may assume that $\mathscr{M}_{i_{0}}^{\cdot}$ is a complex of free modules over $Y^{\left(i_{0}\right)}$. Then the modules above are isomorphic to $\lim _{\longrightarrow} H^{k}\left(\mathscr{L}_{i}^{*}\right)$, resp. $H^{k}\left(\overline{\mathscr{L}}^{*}\right)$, where

$$
\mathscr{L}_{i}^{\cdot}=\mathscr{H}_{o m_{Y^{(i)}}}\left(\mathscr{M}_{i}^{\cdot}, \mathscr{N}_{i}\right)_{y_{0}} \cong \mathscr{L}_{i_{0}}^{\cdot} \otimes_{A^{\left(i_{0}\right)}} A^{(i)},
$$

resp.

$$
\overline{\mathscr{L}}^{\cdot}=\mathscr{\mathscr { L }} o m_{\bar{Y}}\left(\overline{\mathscr{M}}^{\cdot}, \overline{\mathscr{N}}\right)_{y_{0}} \cong \mathscr{L}_{i_{0}} \otimes_{A^{\left(i_{0}\right)}} \bar{A} .
$$

Now (5.5) follows from the fact that $\lim _{\longrightarrow}$ is an exact functor.

We note two important special cases of (5.4). In the first one, we apply (5.4) to $\mathscr{F}_{i_{0}}=\mathscr{O}_{X^{\left(i_{0}\right)}}=\mathscr{G}_{i_{0}}$ and obtain 
(5.6) Corollary. With the notation as in (5.3), let $\mathscr{N}_{i_{0}}$ be a coherent $\mathscr{O}_{Y^{\left(i_{0}\right)}}$ module and set $\mathscr{N}_{i}:=p_{i i_{0}}^{*}\left(\mathscr{N}_{i_{0}}\right)$ and $\overline{\mathscr{N}}:=p_{i_{0}}^{*}\left(\mathscr{N}_{i_{0}}\right), i \geq i_{0}$. Assume that $f^{\left(i_{0}\right)}$ is flat. Then the canonical map

$$
\stackrel{\lim }{\longrightarrow} f_{*}^{(i)}\left(f^{(i) *}\left(\mathscr{N}_{i}\right)\right)_{y_{0}} \rightarrow \bar{f}_{*}\left(\bar{f}^{*}(\overline{\mathscr{N}})\right)_{y_{0}}
$$

is bijective.

(5.7) Corollary. With the notations as in (5.3), assume that $f^{\left(i_{0}\right)}$ is flat and let $\mathscr{N}_{i_{0}}, \mathscr{N}_{i}, \overline{\mathscr{N}}$ be as above. Then the natural morphism

$$
\stackrel{\lim }{\longrightarrow} \mathscr{T}^{k}\left(X^{(i)} / Y^{(i)} ; f^{(i) *}\left(\mathscr{N}_{i}\right)\right)_{y_{0}} \rightarrow \mathscr{T}^{k}\left(\bar{X} / \bar{Y} ; \bar{f}^{*}(\overline{\mathscr{N}})\right)_{y_{0}}
$$

is bijective.

Proof. This follows from (5.4) applied to $\mathscr{F}_{i_{0}}^{\cdot}:=L_{X^{\left(i_{0}\right)} / Y^{\left(i_{0}\right)}}$. Observe that $L \tilde{p}_{i i_{0}}^{*}\left(\mathscr{F}_{i_{0}}^{\bullet}\right)=L_{X^{(i)} / Y^{(i)}}^{\cdot}$ and $L \tilde{p}_{i_{0}}^{*}\left(\mathscr{F}_{i_{0}}^{*}\right)=L_{\bar{X} / \bar{Y}}^{\cdot}($ see $(3.16))$.

Another important application of (5.1) is

(5.8) Proposition. Let $f: X \rightarrow Y$ be a morphism of complex s-spaces and $\mathscr{F}, \mathscr{G} \in \operatorname{Coh}(X)$ such that $\mathscr{G}$ is flat and proper over $Y$. Let $L f_{\#}$ be as in (5.1) and let $f_{\#}(\mathscr{F}):=H^{\circ}\left(L f_{\#}(\mathscr{F})\right)$. Then, for every $\mathscr{N} \in \operatorname{Coh}(Y)$, there is an isomorphism

$$
f_{*}\left(\mathscr{H} \operatorname{om}_{X}\left(\mathscr{F}, \mathscr{G} \otimes f^{*}(\mathscr{N})\right)\right) \cong \mathscr{H} o m_{Y}\left(f_{\#}(\mathscr{F}), \mathscr{N}\right),
$$

which is functorial in $\mathscr{F}$ and $\mathscr{N}$. Moreover, $f_{\#}$ is compatible with arbitrary base change $Y^{\prime} \rightarrow Y$.

Proof (see also $\left.\left[\mathrm{F}_{2}\right]\right)$. Since $\operatorname{Ext}_{f}^{k}\left(\mathscr{F}, \mathscr{G} \otimes f^{*}(\mathscr{N})\right)$ vanishes for $k<0$, the cohomology of $L f_{\#}(\mathscr{F})$ must be concentrated in nonnegative degrees. This and (5.1) proves (5.8).

(5.9) Proposition. Let $S$ be a complex s-space and $\mathscr{M} \in \operatorname{Coh}(S)$. Then the functor

$$
F: \text { s-An } / S \rightarrow \text { sets }
$$

with $F(T):=\mathscr{H}_{0} m_{T}\left(\mathscr{M}_{T}, \mathscr{O}_{T}\right)_{\mathrm{ev}}$ is representable by a complex s-space over $S$.

The space representing $F$ will always be denoted by $\mathbb{V}(\mathscr{M})$. Clearly, $\mathscr{M} \rightarrow$ $\mathbb{V}(\mathscr{M})$ is a contravariant functor. We call $\mathbb{V}(\mathscr{M})$ the linear fiber space associated to $\mathscr{M}$.

Proof. It is sufficient to construct $\mathbb{V}(\mathscr{M})$ locally in $S$. Therefore we may assume that there is a presentation

$$
\mathscr{O}_{S}^{a_{1}} \times \mathscr{O}_{S}^{b_{1}} \underset{\mathfrak{a}}{\rightarrow} \mathscr{O}_{S}^{a_{0}} \times \mathscr{O}_{S}^{b_{0}} \rightarrow \mathscr{M} \rightarrow 0
$$

where the generators of $\mathscr{\sigma}_{S}^{a_{i}}$, resp. $\mathscr{\sigma}_{S}^{b_{i}}$, are concentrated in even, resp. odd, degree. The matrix $\mathfrak{a}$ induces a map

$$
S \times \mathbb{C}^{a_{0} \mid b_{0}} \underset{f}{\rightarrow} S \times \mathbb{C}^{a_{1} \mid b_{1}}
$$

and the reader may easily verify that, similarly as in the classical case, $f^{-1}(S \times 0)$ represents $F$.

This proposition allows us to generalize $\left[F_{2},(3.2)\right.$ and (3.3)] to the case of complex s-spaces. 
(5.10) Corollary. Let $f, \mathscr{F}, \mathscr{G}$ be as in (5.8). Then the functor $F: \mathrm{s}-\mathrm{An} / Y \rightarrow$ sets given by

$$
F(T):=\mathscr{H} o m_{X_{T}}\left(\mathscr{F}_{T}, \mathscr{G}_{T}\right)_{\mathrm{ev}}
$$

is representable by a linear fiber space over $Y$.

Proof. With the notation as in (5.8), we have that $F(T)$ is canonically isomorphic to $\mathscr{H}_{0} m_{T}\left(f_{\#}(\mathscr{F})_{T}, \mathscr{O}_{T}\right)_{\text {ev }}$. Hence $F$ is represented by $\mathbb{V}\left(f_{\#}(\mathscr{F})\right)$.

The functor $F$ is also denoted by $\mathscr{M}_{o r}(\mathscr{F}, \mathscr{G})$ whereas the space representing $F$ is denoted by $\operatorname{Mor}_{Y}(\mathscr{F}, \mathscr{G})$

(5.11) Corollary. Let $f, \mathscr{G}$ be as in (5.8) and suppose that $\overline{\mathscr{G}}$ is a coherent quotient of $\mathscr{G}$. Then the equality functor $\operatorname{Equ}(\mathscr{G}, \bar{G})$ on $\mathrm{s}-\mathrm{An} / Y$ given by

$$
\operatorname{Equ}(\mathscr{G}, \overline{\mathscr{G}})(T):= \begin{cases}\{1\} & \text { if } \mathscr{G}_{T}=\overline{\mathscr{G}}_{T}, \\ \varnothing & \text { if } \mathscr{G}_{T} \neq \overline{\mathscr{G}}_{T}\end{cases}
$$

is representable by a closed subspace of $Y$.

Proof. Set $\mathscr{F}=\operatorname{ker}(\mathscr{G} \rightarrow \overline{\mathscr{G}})$. The morphism $\mathscr{F} \rightarrow \mathscr{G}$ gives rise to a section $Y \stackrel{\sigma}{\rightarrow} \operatorname{Mor}_{Y}(\mathscr{F}, \mathscr{G})$. If $Y \hookrightarrow \operatorname{Mor}_{Y}(\mathscr{F}, \mathscr{G}) \cong \mathbb{V}\left(f_{\#}(\mathscr{F})\right)$ is the zero section, then obviously $\operatorname{Equ}(\mathscr{G}, \overline{\mathscr{G}})$ is represented by $\sigma^{-1}(Y)$.

In the classical case, (5.11) was obtained by Pourcin [Po].

\section{FORMAL DEFORMATION THEORY OF COMPLEX S-SPACES}

The purpose of this section is to develop the deformation theory in supergeometry along the lines of $\left[\mathrm{Ri}, \mathrm{F}_{3}\right.$, Schu].

(6.1) We remind the reader of the notation of fibered groupoid (see [Ri]). Let $\mathscr{C}$ be a category. By a fibered groupoid over $\mathscr{C}$ we mean a category $\mathscr{F}$ and a covariant functor $p: \mathscr{F} \rightarrow \mathscr{C}$ such that the following three properties are satisfied.

(1) For every morphism $f: X \rightarrow Y$ in $\mathscr{C}$ and every object $a$ in $\mathscr{F}$ over $Y$ there is a cartesian morphism $\tilde{f}: b \rightarrow a$ with $p(\tilde{f})=f$ (i.e., for every morphism $c \stackrel{\tilde{\hat{f}}}{\rightarrow} a$ over $f$ in $\mathscr{F}$ there is a unique morphism $c \stackrel{g}{\rightarrow} b$ over $\operatorname{id}_{X}$ satisfying $\tilde{f} \circ g=\tilde{\tilde{f}}$ ).

(2) Compositions of cartesian morphisms are cartesian.

(3) $\tilde{f}: a \rightarrow b$ is an isomorphism iff $p(\tilde{f})$ is.

The object $b$ in (1) will often be denoted by $p^{*}(a)$ or $a \times_{Y} X$.

Similarly, a cofibered groupoid is a functor $p: \mathscr{F} \rightarrow \mathscr{C}$ such that the dual functor $p^{0}: \mathscr{F}^{0} \rightarrow \mathscr{C}^{0}$ is a fibered groupoid. In a cofibered groupoid, the cofiber product will be denoted by $a \otimes_{A} B$ or $f_{*}(a)$ if $f: A \rightarrow B$ is a morphism with $p(a)=A$. Assume that $p: \mathscr{F} \rightarrow \mathscr{C}$ is a fibered or cofibered groupoid and $X$ is an object of $\mathscr{C}$. Then the subcategory of $\mathscr{F}$ consisting of objects (resp. morphisms) over $X$ (resp. id ${ }_{X}$ ), will be denoted by $\mathscr{F}(X)$.

(6.2) In the following, the basis category $\mathscr{C}$ will be the category s-An/S or $s \mathscr{C}_{\wedge}$. If $p: \mathscr{F} \rightarrow \mathrm{s}$-An $/ S$ is a fibered groupoid and $s \in S$ is fixed then $p$ induces in a natural way a fibered groupoid over the category of germs of complex s-spaces by setting $\mathscr{F}\left(T, t_{0}\right):=\lim \mathscr{F}(U)$, where $U$ runs through the neighborhoods of $t_{0}$ in $T$. Dually, we obtain a cofibered groupoid

$$
{ }_{s} p: \widetilde{\mathscr{F}} \rightarrow s \mathscr{C}_{\wedge}, \quad \wedge:=\mathscr{O}_{S, s} .
$$


On the other hand, if $p: \mathscr{F} \rightarrow s \mathscr{C}_{\wedge}$ is a cofibered groupoid then $p$ induces in a natural way a cofibered groupoid $\hat{p}: \widehat{\mathscr{F}} \rightarrow s \hat{\mathscr{C}}_{\wedge}$ (see [Ri, p. 9]).

(6.3) Let $p: \mathscr{F} \rightarrow s \mathscr{C}_{\wedge}$ be a cofibered groupoid. An object $a \in \mathscr{F}(A)$ will be called versal if the following lifting property is satisfied.

(V) Let $b \stackrel{f}{\rightarrow} b^{\prime}$ be a morphism in $\mathscr{F}$ such that $p(f): B \rightarrow B^{\prime}$ is surjective. Then every morphism $a \rightarrow b^{\prime}$ can be lifted to a morphism $a \rightarrow b$.

We also call $(A, a)$ the versal deformation of $a_{0}:=a \otimes_{A} \mathbb{C}$. Similarly, $a$ is called formally versal if the lifting property $(\mathrm{V})$ is satisfied in the case that $B$ and $B^{\prime}$ are artinian. The notion of formal versality also makes sense for $\bar{a} \in \widehat{\mathscr{F}}(\bar{A}), \bar{A} \in s \hat{\mathscr{C}}_{\wedge} . \quad a$ is called (formally) semiuniversal if it is (formally) versal and if in (V) the morphism $A \rightarrow B$ induced by $a \rightarrow b$ is unique modulo $\mathfrak{m}_{B}^{2}$.

If dually $p: \mathscr{F} \rightarrow \mathrm{s}-\mathrm{An} / S$ is a fibered groupoid and $a \in \mathscr{F}(T)$ then $a$ is said to be versal (formally versal) at $t \in T$ if the same is true for $a$, regarded as objects in ${ }_{s} \widetilde{F}\left(\mathscr{O}_{T, t}\right)$ (see (6.2)). Here $s$ denotes the image of $t$ under the structure morphism $T \rightarrow S$.

The following three examples will turn out to be important in later sections.

(6.4) (1) (Deformations of compact complex s-spaces) Let $p: \mathscr{F} \rightarrow$ s-An be the groupoid consisting of flat proper morphisms $a=(X \rightarrow T)$ of complex sspaces; here $p(a):=T$. A morphism $a \rightarrow b=(Y \rightarrow S)$ consists of a cartesian diagram

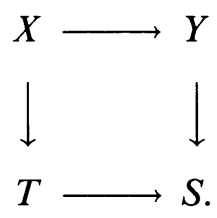

(2) (Deformations of modules) Let $f: X \rightarrow S$ be a fixed morphism of complex s-spaces. An object of $\mathscr{F}$ consists of a pair $(\mathscr{M}, T)$, where $T \in \mathrm{s}$-An $/ S$ and $\mathscr{M}$ is a coherent $\mathscr{O}_{X_{T}}$-module which is proper and flat over $T$. If $\left(\mathscr{M}^{\prime}, T^{\prime}\right)$ is another object of $\mathscr{F}$ then a morphism $(\mathscr{M}, T) \rightarrow\left(\mathscr{M}^{\prime}, T^{\prime}\right)$ in $\mathscr{F}$ consists of a morphism $T \stackrel{\varphi}{\rightarrow} T^{\prime}$ of s-spaces and an isomorphism $\left(1_{X} \times_{S} \varphi\right)^{*}\left(\mathscr{M}^{\prime}\right) \stackrel{\sim}{\rightarrow} \mathscr{M}$.

(3) (Deformations of quotients) Let $f: X \rightarrow S$ be a morphism of complex s-spaces and $\mathscr{M} \in \operatorname{Coh}(X)$. Let $\mathscr{F}$ be the category of all pairs $(\mathscr{G}, T)$, where $T \in s-\mathrm{An} / S$ and $\mathscr{G}$ is a quotient of $\mathscr{M}_{T}$, being proper and flat over $T$. A morphism $(\mathscr{G}, T) \rightarrow\left(\mathscr{G}^{\prime}, T^{\prime}\right)$ in $\mathscr{F}$ consists of a morphism $\varphi: T \rightarrow T^{\prime}$ with the property that $\left(1_{X} \times_{S} \varphi\right)^{*}\left(\mathscr{G}^{\prime}\right)=\mathscr{G}$ as quotients of $\mathscr{M}_{T}$.

(6.5) Let $p: \mathscr{F} \rightarrow \mathrm{s}-\mathrm{An} / S$ be a fibered groupoid. For an object $a$ of $\mathscr{F}$, let $\mathscr{F}_{a}$ denote the category of all morphisms $a \rightarrow b$ in $\mathscr{F}$ where the morphisms in $\mathscr{F}_{a}$ are given by commutative diagrams:

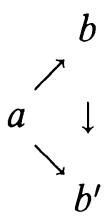


Moreover, an overbar will always indicate the set of isomorphism classes of a category, i.e., $\overline{\mathscr{F}}(T), \overline{\mathscr{F}}_{a}(T), \ldots$ is the set of isomorphism classes of $\mathscr{F}(T)$, $\mathscr{F}_{a}(T), \ldots$ If $T \in \mathrm{s}$-An $/ S$ and $\mathscr{M}$ is a coherent $\mathscr{O}_{T}$-module then we put $D_{a}(\mathscr{M}):=\overline{\mathscr{F}}_{d}(T[\mathscr{M}])$ if $a \in \mathscr{F}(T)$. The sheaf associated to the presheaf $U \mapsto D_{a \mid U}(\mathscr{M} \mid U)$ on $T$ will be denoted by $\mathscr{D}_{a}(\mathscr{M})$. Dually, if $p: \mathscr{F} \rightarrow s \mathscr{C}_{\wedge}$ is a cofibered category then $\mathscr{F}_{a}$ will denote the category of all arrows $b \rightarrow a$ in $\mathscr{F}$. Again, an overbar will indicate the set of isomorphism classes. For a finite $A$-module $\mathscr{M}$ we set $D_{a}(M):=\overline{\mathscr{F}}_{a}(A[M])$ if $A=p(a)$.

(6.6) We are now able to introduce the Schlessinger conditions.

(S1)(a) Let $T \stackrel{\beta}{\rightarrow} T^{\prime}, T \stackrel{\alpha}{\rightarrow} Y$ be morphisms in s-An/S such that $\beta$ is an extension and $T_{\mathrm{rd}} \rightarrow Y$ is a closed embedding. Then, for $a \in \mathscr{F}(T)$ the canonical map

$$
\overline{\mathscr{F}}_{a}\left(T^{\prime} \amalg_{T} Y\right) \rightarrow \overline{\mathscr{F}}_{a}\left(T^{\prime}\right) \times \overline{\mathscr{F}}_{a}(Y)
$$

is surjective.

(b) Let $T \hookrightarrow T^{\prime}$ be a closed embedding and $\mathscr{M}$ a coherent $\mathscr{O}_{T_{\mathrm{rd}}}$-module. Then the canonical map

$$
D_{a^{\prime}}(\mathscr{M}) \rightarrow D_{a}(\mathscr{M})
$$

is bijective.

Clearly, (S1) follows from the following slightly stronger condition:

$(\mathrm{S} 1)^{\prime}$ With the notation as in $(\mathrm{S} 1)(\mathrm{a})$, the functor

$$
\mathscr{F}_{a}\left(T^{\prime} \amalg_{T} Y\right) \rightarrow \mathscr{F}_{a}(T) \times \mathscr{F}_{a}(Y)
$$

is an equivalence of categories.

Condition (S2) of Schlessinger must be formulated as follows:

(S2) If $a \in \mathscr{F}(T)$ and $\mathscr{M}$ is a coherent $\mathscr{O}_{T_{\mathrm{rd}}}$-module then $\mathscr{D}_{a}(\mathscr{M})$ is also a coherent $\mathscr{O}_{T_{\text {rd }}}$-module.

The following lemma is easily deduced from $\left[\mathrm{Schu}_{2}\right]$ :

(6.7) Lemma. In the examples (6.4)(1)-(3), property $(\mathrm{S} 1)^{\prime}$ is fulfilled.

(6.8) If $p: \mathscr{F} \rightarrow s \mathscr{C}_{\wedge}$ is a cofibered groupoid then one can also formulate conditions (S1) and (S1) in a dual way. We leave the straightforward formulation to the reader. We will say that $(\mathrm{S} 1),(\mathrm{S} 1)^{\prime},(\mathrm{S} 2)$ are satisfied for artinian bases if this property holds in at least the case that all rings occurring in (S1), (S1)', $(\mathrm{S} 2)$, resp., are artinian.

(6.9) Lemma. Let $p: \mathscr{F} \rightarrow \mathrm{s}-\mathrm{An} / S$ be a fibered groupoid satisfying (S1) and $a \in \mathscr{F}(T)$. Then the following hold:

(1) The functors $D_{a}(-)$ and $\mathscr{D}_{a}(-)$ are half exact on $\operatorname{Coh}\left(T_{\mathrm{rd}}\right)$.

(2) If $\mathscr{M} \in \operatorname{Coh}\left(T_{\text {rd }}\right)$, then $D_{a}(\mathscr{M})$ carries in a natural way the structure of a $\Gamma\left(T, \mathscr{O}_{T_{\mathrm{rd}}}\right)$-module.

Proof. For the proof of $(1)$, see $\left[\mathrm{F}_{3},(2.1)\right]$. That $\mathscr{D}_{a}(\mathscr{M})$ has a natural $\Gamma\left(T, \mathscr{O}_{T_{\text {rd }}}\right)$-module structure follows easily from the functoriality and the fact that $D_{a}(\mathscr{M}) \times D_{a}(\mathscr{M}) \cong D_{a}(\mathscr{M} \times \mathscr{M})$ by $(\mathrm{S} 1)$.

(6.10) Let $p: \mathscr{F} \rightarrow \mathrm{s}$-An/ $S$ be a fibered groupoid satisfying (S1). For $a \in \mathscr{F}$, $T=p(a)$ let $g: \mathscr{C}_{a} \rightarrow \operatorname{Coh}\left(T_{\text {rd }}\right)$ be the cofibered groupoid of extensions of $a:$ An object of $\mathscr{C}_{a}$ over $\mathscr{M} \in \operatorname{Coh}\left(T_{\text {rd }}\right)$ is a pair $\left(T^{\prime}, a^{\prime}\right)$, where $T \stackrel{i}{\rightarrow} T^{\prime}$ is 
an extension of $T$ by $\mathscr{M}$ and $a \rightarrow a^{\prime}$ is a morphism over $i$. The morphisms in $\mathscr{C}_{a}$ are defined in an obvious way (see $\left[\mathrm{F}_{3}\right.$, p. 454]). We set

$$
\operatorname{Ex}(a, \mathscr{M}):=\overline{\mathscr{C}}_{a}(\mathscr{M}) \text {. }
$$

The pairs $\left(T^{\prime}, a^{\prime}\right)$ as above will be called extensions of $a$ by $\mathscr{M}$. Because of (S1), the canonical map

$$
\operatorname{Ex}(a, \mathscr{M} \times \mathscr{N}) \rightarrow \operatorname{Ex}(a, \mathscr{M}) \times \operatorname{Ex}(a, \mathscr{N})
$$

is surjective. If $(\mathrm{S} 1)^{\prime}$ is fulfilled then this map is even bijective and $\operatorname{Ex}(a, \mathscr{M})$ carries a natural $\Gamma\left(T, \mathscr{O}_{T}\right)$-module structure.

In the general case, $\operatorname{Ex}(a, \mathscr{M})$ has no module structure. But nevertheless there is a 0 -element given by the trivial extension $T \hookrightarrow T[\mathscr{M}]$ and $a[\mathscr{M}]:=$ $p^{*}(a)$ if $p: T[\mathscr{M}] \rightarrow T$ is the natural map. Just as in $\left[\mathrm{F}_{3}\right.$, p. 454], we can define canonical maps

$$
\begin{aligned}
c & : \operatorname{Der}_{S}\left(\mathscr{O}_{T}, \mathscr{M}\right)_{\mathrm{ev}} \rightarrow D_{a}(\mathscr{M}), \\
i & : D_{a}(\mathscr{M}) \rightarrow \operatorname{Ex}(a, \mathscr{M}), \\
\pi & : \operatorname{Ex}(a, \mathscr{M}) \rightarrow \operatorname{Ex}(T / S, \mathscr{M}),
\end{aligned}
$$

where $\operatorname{Ex}(T / S, \mathscr{M})$ is the set of isomorphism classes of extensions of $T / S$ by $\mathscr{M}$. The proof of (1) and (2) in the following result is the same as in $\left[\mathrm{F}_{3},(2.2)\right]$.

(6.11) Lemma. (1) $\pi(\operatorname{Ex}(a, \mathscr{M})) \subseteq \operatorname{Ex}(T / S, \mathscr{M})$ is a $\Gamma\left(T, \mathscr{O}_{T}\right)$-submodule.

(2) $\pi \circ i=0, i \circ c=0$, and $\operatorname{Coker}(c) \rightarrow \pi^{-1}(0)$ is bijective.

We remark that in the case that $\operatorname{Ex}(a, \mathscr{M})$ carries a module structure, (6.11) means that

$$
\operatorname{Der}_{S}\left(\mathscr{O}_{S}, \mathscr{M}\right)_{\mathrm{ev}} \stackrel{c}{\rightarrow} D_{a}(\mathscr{M}) \stackrel{i}{\rightarrow} \mathrm{Ex}(a, \mathscr{M}) \stackrel{\pi}{\rightarrow} \mathrm{Ex}(T / S, \mathscr{M})
$$

is an exact sequence.

Also for the proof of the following two results we refer the reader to $\left[\mathrm{F}_{3}\right.$, (3.1) and (3.2)].

(6.12) Lemma. Assume that $p: \mathscr{F} \rightarrow \mathrm{s}$-An/S satisfies (S1) and (S2). Let $a$ be in $\mathscr{F}(T)$ and consider a coherent $\mathscr{O}_{T_{\mathrm{rd}}}$-module $\mathscr{M}$ and a coherent ideal $\mathfrak{a} \subseteq \mathscr{O}_{T_{\mathrm{rd}}}$. If $t \in V(\mathfrak{a})$, let $p_{t}$ denote the canonical map

$$
p_{t}: \mathscr{E}_{x}(a, \mathscr{M})_{t} \rightarrow \underset{n}{\lim } \mathscr{E}_{x}\left(a, \mathscr{M} / \mathfrak{a}^{n} \mathscr{M}\right)_{t} .
$$

Then $p_{t}^{-1}(0)=0$.

Here $\mathscr{E}_{x}(a, \mathscr{M})$ denotes the sheaf associated to the presheaf

$$
U \mapsto \mathscr{E}_{x}(a|U, \mathscr{M}| U) .
$$

(6.13) Proposition. Assume that (S1) and (S2) are satisfied. Then the following statements are equivalent.

(1) $a$ is formally versal in $t$.

(2) $\mathscr{E}_{x}\left(a, \mathbb{C}_{t} x+\mathbb{C}_{t} \xi\right)_{t}=0$.

(3) $\mathscr{E}_{x}(a, \mathscr{M})_{t}=0$ for every coherent $\mathscr{O}_{T_{\mathrm{rd}}}$-module $\mathscr{M}$.

Here $\mathbb{C}_{t} x+\mathbb{C}_{t} \xi$ shortly denotes the $\mathscr{O}_{T}$-module concentrated in $t$ whose even, resp. odd, part is $\mathbb{C}_{t} x$, resp. $\mathbb{C}_{t} \xi$. For the proof, see $\left[\mathrm{F}_{3},(3.2)\right]$. 
In the next result we sketch the proof of Schlessinger's theorem in the supercase. Let $p: \mathscr{F} \rightarrow s \mathscr{C}_{\wedge}$ be a cofibered groupoid and $a \in \mathscr{F}(A)$. We will say that $a$ is versal up to level $l$ if condition (V) in (6.3) is satisfied in the special case that $\mathfrak{m}_{B}^{l+1}=0$.

(6.14) Theorem. Let $p: \mathscr{F} \rightarrow s \mathscr{C}_{\wedge}$ be a cofibered groupoid which satisfies (S1) and (S2) for artinian algebras. Then every $a_{0} \in \mathscr{F}(\mathbb{C})$ admits a formally versal deformation $\bar{a} \in \widehat{\mathscr{F}}(\bar{A})$.

Proof. We proceed similarly as in the classical case (see [Ri, p. 12]). Let $t, \ldots, t_{n}, \tau_{1}, \ldots, \tau_{m} \in D_{a_{0}}(\mathbb{C} x+\mathbb{C} \xi)$ be a basis with $\operatorname{deg}\left(t_{i}\right)=0, \operatorname{deg}\left(\tau_{j}\right)=1$. By using (S1) we get a deformation $a_{1} \in D_{a_{0}}(V)$ with $V:=\bigoplus_{i=1}^{m} \mathbb{C} x_{i} \times$ $\bigoplus_{j=1}^{m} \mathbb{C} \xi_{j}$ such that $a_{1}$ is versal up to level 1 . Here $a_{1}$ is the element of $D_{a_{0}}(V)$ corresponding to the tuple $\left(t_{1}, \ldots, t_{n}, \tau_{1} \ldots, \tau_{m}\right)$ under the isomorphism

$$
D_{a_{0}}(V) \cong \bigoplus_{i} D_{a_{0}}\left(\mathbb{C} x_{i}\right) \times \bigoplus_{j} D_{a_{0}}\left(\mathbb{C} \xi_{j}\right) \text {. }
$$

Now assume that $\left(A_{l}, a_{l}\right)$ is already constructed such that $a_{l}$ is versal up to level $l$ and $A_{l}$ is a quotient of the free power series ring

$$
\bar{R}:=\widehat{\Lambda} \llbracket x_{1}, \ldots, x_{n} ; \xi_{1}, \ldots, \xi_{m} \rrbracket .
$$

By using (S1) it is seen that there is a unique ideal $\mathfrak{a}_{l+1}$ of $\bar{R}$ which is maximal among the $\mathbb{Z}_{2}$-graded ideals $\mathfrak{b} \subseteq \bar{R}$ such that (a) $\mathfrak{m}_{\bar{R}}^{l+2} \subseteq \mathfrak{b}$, (b) $\mathfrak{a}_{l} \supseteq \mathfrak{b}$, and (c) there is a $b \in \mathscr{F}(\bar{R} / \mathfrak{b})$ inducing $a_{l}$ on $\mathscr{F}\left(\bar{R} / \mathfrak{a}_{l}\right)$. Now with similar arguments as in [Ri] it is easily shown that $\bar{A}:=\lim R / \mathfrak{a}_{l}, \bar{a}:=\lim _{\longleftarrow} a_{l}$ is formally versal.

(6.15) Let $p: \mathscr{F} \rightarrow \mathrm{s}-\mathrm{An} / S$ be a fibered groupoid satisfying (S1) and (S2) (see (6.6)). If $a \in \mathscr{F}(T)$ and $\mathscr{M}$ is a coherent $\mathscr{O}_{T_{\mathrm{rd}}}$-module then we denote by $\mathscr{O}_{a}(\mathscr{M})$ the quotient sheaf $\mathscr{E} \times(T / S, \mathscr{M}) / \mathscr{E} \times(a, \mathscr{M})$, which by $(6.11)$ is a $\mathscr{O}_{T_{\mathrm{rd}}}$-module. Obviously, $\mathscr{O}_{a}(\mathscr{M})$ is functorial in $\mathscr{M}$. We now introduce the conditions (S3) and (S4).

(S3) For all $a, T, \mathscr{M}$ as above, $\mathscr{O}_{a}(\mathscr{M})$ is coherent.

(S4) Let $a, T, \mathscr{M}$ be as above and suppose that $T^{\prime} \subseteq T$ is an irreducible reduced subspace. Then there is a Zariski-open dense subset $V^{\prime} \subseteq T^{\prime}$ such that for $t \in V^{\prime}$ the canonical morphism

(a) $\mathscr{D}_{a}\left(\mathscr{O}_{T^{\prime}} x \oplus \mathscr{O}_{T^{\prime}} \xi\right) \otimes \mathscr{O}(t)_{t} \rightarrow \mathscr{D}_{a}(\mathscr{O}(t) x \oplus \mathscr{O}(t) \xi)_{t}$ is bijective and

(b) $\mathscr{O}_{a}\left(\mathscr{O}_{T^{\prime}} x \oplus \mathscr{O}_{T^{\prime}} \xi\right) \otimes \mathscr{O}(t)_{t} \rightarrow \mathscr{O}_{a}(\mathscr{O}(t) x \oplus \mathscr{O}(t) \xi)_{t}$ is injective.

Here $x$, resp. $\xi$, is a basis element of even, resp. odd, degree. We are now able to formulate a criterion for the openness of versality.

(6.16) Theorem. Suppose that $p: \mathscr{F} \rightarrow \mathrm{s}-\mathrm{An} / S$ is a fibered groupoid satisfying (S1), (S2), (S3), and (S4). Then, for every $a \in \mathscr{F}(T)$, the set of points $t \in T$ in which $a$ is formally versal is Zariski-open.

We remark that the proof of $\left[\mathrm{F}_{3}, \mathrm{pp} .458-459\right]$ can be carried over to the case of superspaces with the obvious changes. Similarly, the proof of the following result is the same as for even spaces (see [loc. cit., (5.3)]).

(6.17) Proposition. Assume that $\bar{b}$ is formally semiuniversal in $\widehat{\mathscr{F}}$ and that $\bar{b} \rightarrow \bar{a}$ is a morphism such that $\bar{a}$ is formally versal. Then $\bar{A}:=p(\bar{a})$ is a formal 
power series ring over $\bar{B}:=p(b)$, i.e., $\bar{A} \cong \bar{B} \llbracket X_{1}, \ldots, X_{n} ; Y_{1}, \ldots, Y_{m} \rrbracket$, where the $X_{i}$ and $Y_{i}$ are generators of even, resp. odd, degree.

(6.18) In the rest of this section, we will verify that in the examples (6.4) the criteria above are satisfied. In order to do this, we need the notion of obstruction theory. Assume that for every $a \in \mathscr{F}(T)$ there is a functor

$$
\mathscr{O} b_{a}: \operatorname{Coh}(T) \rightarrow \operatorname{Coh}(T)
$$

for which condition (S4)(b) is satisfied. If there is a functorial map $\mathscr{O}_{a}(M) \rightarrow$ $\mathscr{O} b_{a}(M)$ which is injective, then obviously (S3) and (S4)(b) are also fulfilled.

We are now able to prove:

(6.19) Proposition. For deformations of compact complex s-spaces (see (6.4)(1)) formal versality is open.

Proof. From $\S 4$ we obtain that for a proper morphism $a=(X \stackrel{f}{\rightarrow} S)$ the module $\mathscr{D}_{a}(\mathscr{M})$ is canonically isomorphic to the sheaf associated to the presheaf $S \supseteq U \mapsto T_{f^{-1}(U) / U^{\prime}}^{1}\left(f^{*} \mathscr{M}\right)$, i.e., $\mathscr{D}_{a}(\mathscr{M}) \cong \mathscr{E} x t_{f}^{1}\left(L_{X / S}^{\cdot}, f^{*} \mathscr{M}\right)$. Similarly, an obstruction theory is given by the sheaf associated to $U \mapsto T_{f^{-1}(U) / U}^{2}\left(f^{*}(\mathscr{M})\right)$ which is just $\mathscr{E} x t_{f}^{2}\left(L_{X / S}^{\bullet}, f^{*} \mathscr{M}\right)$. Hence (S3) is satisfied. By using (5.1) it is also easily seen that (S4) is satisfied. This proves (6.19).

(6.20) Proposition. In the examples (6.4)(2), (3), formal versality is open.

The proof of this result is similar as above (see $\left[\mathrm{F}_{1}, \mathrm{Bi}_{2}\right]$ for the classical case).

\section{REPRESENTABILITY CRITERIA}

Let $\wedge$ be an analytic s-algebra and consider a functor $F: s \mathscr{C}_{\wedge} \rightarrow$ sets as in (1.12). The following result is the generalization of a result of Bingener $\left[\mathrm{Bi}_{1}\right.$, (2.4)] to supergeometry.

(7.1) Theorem. Let $F$ satisfy the (L1)-condition as above (see (1.12)). Suppose that there exists a formal versal deformation $(\bar{A}, \bar{a})$ of $a_{0} \in F\left(\wedge / \mathfrak{m}_{\wedge}\right)$ with $\bar{A} \in s \hat{\mathscr{C}}_{\wedge}$. Then

(1) There exists an analytic s-algebra $A \in s \mathscr{C}_{\wedge}$ and $a \in F(A)$ such that $(\widehat{A}, \hat{a})$ is $\wedge$-isomorphic to $(\bar{A}, \bar{a})$.

(2) If $F$ satisfies (L2) then $(A, a)$ is uniquely determined.

Proof. The proof of (2) is exactly as in $\left[\mathrm{Bi}_{1},(2.4)(2)\right]$. For the proof of (1), we may assume with the same argument as in [loc. cit., p. 326, first section in the proof of (2.4)] that $\wedge=\mathbb{C}$. There is a finite injective $\mathbb{C}$-morphism

$$
\widehat{P} \rightarrow \bar{A}, \quad P:=\mathbb{C}\left\{X_{1}, \ldots, X_{n}\right\},
$$

where the $X_{i}$ are variables of even degree. Set $\bar{A}^{*}:=\bar{A}_{0}\left[\bar{A}_{1}\right]$, where we consider $\bar{A}^{*}$ as an algebra via $\bar{A}_{1}^{2}=0$. Then $\bar{A}^{*}$ is an even complete $\mathbb{C}$-algebra. After changing the $X_{i}$ suitably we may assume that, with the notation as in $\left[\mathrm{Bi}_{1}, \S 1\right]$, the $I_{\hat{p}}^{\mu}\left(\bar{A}^{*}\right), 0 \leq \mu \leq n$, have a preparation over $A$. Let $\mathscr{C}_{P}$ denote the category of all even analytic $P$-algebras. For $P^{\prime} \in \mathscr{C}_{P}$, we consider tuples $\left(A^{\prime} ; J^{\nu} \rightarrow A^{\prime *}, 0 \leq \nu \leq n ; a^{\prime}\right)$, where $A^{\prime}$ is a $P^{\prime}$-s-algebra, $a^{\prime} \in F\left(A^{\prime}\right), A^{\prime *}:=A_{0}^{\prime}\left[A_{1}^{\prime}\right]$, and $\left(\bar{A}^{\prime *}, J^{\nu} \rightarrow A^{\prime *}\right) \in G\left(P^{\prime}\right)$, where $G$ is defined 
as in $\left[\mathrm{B}_{1},(1.5)\right]$. These tuples form in a natural way a category $H\left(P^{\prime}\right)$, and a morphism of $P$-algebras $P^{\prime} \rightarrow P^{\prime \prime}$ induces a natural functor $H\left(P^{\prime}\right) \rightarrow H\left(P^{\prime \prime}\right)$. Let $\bar{H}\left(P^{\prime}\right)$ be the set of isomorphism classes of $H$. Then $\bar{H}$ becomes a functor on $\mathscr{C}_{P}$ with values in the category of sets satisfying (L1) for even analytic algebras, i.e., if $\bar{B} \in \hat{\mathscr{C}}_{P}$ and $\bar{B}=\lim _{\longrightarrow} B^{(i)}, B^{(i)} \in \mathscr{C}_{P}$, then $\lim _{\longrightarrow} \bar{H}\left(B^{(i)}\right) \rightarrow \bar{H}(\widehat{B})$ has dense image. Now we can continue, as in $\left[\mathrm{Bi}_{1},(2.4)\right]$ with this functor $\bar{H}$. Let $N$ be sufficiently large and take an element

$$
\left(A ; J^{\nu} \rightarrow A^{*}, 0 \leq \nu \leq n ; a\right)
$$

in $H(P)$ whose image in $H\left(\widehat{P} / \mathfrak{m}_{\widehat{P}}^{N+1}\right)$ is the same as

$$
\left(\bar{A} ; I_{\widehat{P}}^{\nu}\left(\bar{A}^{*}\right) \rightarrow \bar{A}^{*}, 0 \leq \nu \leq n ; \bar{a}\right) .
$$

An isomorphism between these objects in $H\left(\widehat{P} / \mathfrak{m}_{\hat{p}}^{n+1}\right)$ gives rise to a $\widehat{P}$ morphism of s-algebras $\bar{A} \stackrel{\bar{\psi}}{\rightarrow} A / \mathfrak{m}_{p}^{n+1} A$ mapping $\bar{a}$ onto the image of $a$ in $F\left(A / \mathfrak{m}_{p}^{n+1} A\right)$. Using the formal versality of $(\bar{A}, \bar{a})$ we obtain a lifting $\bar{A} \stackrel{\psi}{\rightarrow} \widehat{A}$ mapping $\bar{a}$ onto $\hat{a}$. By $\left[\mathrm{Bi}_{1},(1.6)\right], \hat{\psi}$ is an isomorphism and we are done. $\S 9$.

We now give two important applications which will turn out to be useful in

(7.2) Let $p: \mathscr{F} \rightarrow s \mathscr{C}_{\wedge}$ be a cofibered groupoid satisfying the Schlessinger conditions $\left(\mathrm{S}^{\prime}\right)$ and $(\mathrm{S} 2)$ (see (6.6) and (6.8)). Let $a_{0} \in F(\mathbb{C})$ be fixed. As in $\S 6$, the groupoid above induces a functor

$$
\overline{\mathscr{F}}_{a_{0}}: s \mathscr{C}_{\wedge} \rightarrow \mathfrak{s e t s},
$$

where $\overline{\mathscr{F}}_{a_{0}}$ is the set of all isomorphism classes of the category $\mathscr{F}_{a_{0}}(A)$. For $a, b \in \mathscr{F}_{a_{0}}(A)$, consider the functor

$$
\text { Iso }_{a_{0}}(a, b): s \mathscr{C}_{A} \rightarrow \text { sets },
$$

where $\operatorname{Iso}_{a_{0}}(a, b)(B)$ is the set of all morphisms $f: a \otimes_{A} B \rightarrow b \otimes_{A} B$ in $\mathscr{F}_{a_{0}}$ (which automatically are isomorphisms). In the case of even complex spaces, the following result is due to Schuster $\left(\right.$ see $\left.\left.\mathrm{Schu}_{1}\right]\right)$ :

(7.3) Proposition. Let $(A, \eta)$ be formally versal, where $A \in s \mathscr{C}_{\wedge}$ and $\eta \in$ $\mathscr{F}_{a_{0}}(A)$. Assume that for all $C \in s \mathscr{C}_{\wedge}$ and $a, b \in \mathscr{F}_{\wedge}(C)$, the functor $\operatorname{Iso}_{a_{0}}(a, b)$ is representable by an analytic $C$-s-algebra. Then $(A, \eta)$ is a versal deformation of $a_{0}$.

Proof. Let $b \rightarrow b^{\prime}$ and $\eta \rightarrow b^{\prime}$ be in $\mathscr{F}_{a_{0}}$ such that the map of underlying analytic s-algebras $B \rightarrow B^{\prime}$ is surjective. We must show that the arrow $\eta \rightarrow b^{\prime}$ can be lifted to an arrow $\eta \rightarrow b$. For this, consider the analytic tensor product $C:=A \widetilde{\otimes}_{\wedge} B$ and $\eta_{C}:=\eta \otimes_{A} C, b_{C}:=b \otimes_{B} C$. Let $D$ represent Iso $a_{a_{0}}\left(\eta_{C}, b_{C}\right)$. The arrow $\eta \rightarrow b^{\prime}$ induces a map $A \rightarrow B^{\prime}$ and so $B^{\prime}$ may be regarded as a $C$-s-algebra. The morphism $\eta \otimes_{A} B^{\prime} \cong \eta_{C} \otimes_{C} B^{\prime} \rightarrow b^{\prime} \cong b_{C} \otimes_{C} B^{\prime}$ gives a map $D \stackrel{\phi}{\rightarrow} B^{\prime}$. Now the arrow $\eta \rightarrow b^{\prime}$ can, at least formally, be lifted to a map $\hat{\eta} \rightarrow \hat{b}$, which gives a formal lifting $\bar{\psi}: \widehat{D} \rightarrow \widehat{B}$ of $\phi$. By Artin's approximation theorem, $\bar{\psi}$ can be approximated by a morphism of $B$-algebras $\psi: D \rightarrow B$ which is again a lifting of $\phi$. Obviously, $\psi$ induces a lifting $\eta \rightarrow b$ of $\eta \rightarrow b^{\prime}$ as desired.

By combining (6.14), (7.1), and (7.3) we obtain: 
(7.4) Corollary. Let the notations be as in (7.2) and assume that the following conditions are satisfied for $a_{0} \in F(\mathbb{C})$ :

(1) $p: \mathscr{F}_{a_{0}} \rightarrow s \mathscr{C}_{\wedge}$ satisfies the Schlessinger conditions for artinian algebras.

(2) For all $C \in s \mathscr{C}_{\wedge}$ and $a, b \in \mathscr{F}_{a_{0}}(C)$ the functor Iso $_{a_{0}}(a, b)$ is representable by an analytic $C$-s-algebra.

(3) The induced functor $\overline{\mathscr{F}_{a_{0}}}$ satisfies the (L1)-condition.

Then $a_{0}$ admits $a$ versal deformation.

In our next result we consider a functor $F: \operatorname{s-An} / S \rightarrow$ sets, where $S$ is a fixed complex s-space.

(7.5) Theorem. Let $F$ be as above and assume that the following conditions are satisfied.

(1) Every $a_{0} \in F(s), s \in S$, admits a semiuniversal deformation.

(2) (Sheaf axiom) For every $T \in \mathrm{s}-\mathrm{An} / S$, the presheaf $T \supseteq U \mapsto F(U)$ on $T$ is a sheaf.

(3) For every $T \in \mathrm{s}-\mathrm{An} / S$ and $a, b \in F(T)$, the set-valued functor $\operatorname{Equ}(a, b)$, with

$$
\operatorname{Equ}(a, b)(Z):= \begin{cases}\{1\} & \text { if } a_{Z}=b_{Z}, \\ \varnothing & \text { otherwise, }\end{cases}
$$

is representable by a locally closed (resp. closed) subspace of $T$.

(4) (Openness of versality) If $T \in \mathrm{s}-\mathrm{An} / S$ and $a \in T$ then the set of points $t \in T$ at which $a$ is formally versal is open in $T$.

Then $F$ can be represented by a not necessarily separated (resp. separated) complex s-space.

$\operatorname{Proof}$ (see also $\left.\left[\mathrm{F}_{2},(9.1)\right]\right)$. Set $I:=\bigcup_{s \in S} F(s)$. For $i=\left(s, a_{0}\right) \in I$, there is a semiuniversal deformation $a_{i}$ of $a_{0}$ over some germ $\left(T_{i}, t_{i}\right)$. In the subsequent Lemma (7.6) we will show that we can choose a representative $T_{i}$ such that $a_{i}$ is universal at every point of $T_{i}$. Now set

$$
X_{0}=\bigcup_{i \in I} T_{i}, \quad a:=\bigcup_{i \in I} a_{i} \in F\left(X_{0}\right)
$$

Observe that $F$ is a sheaf on $X_{0}$. Moreover, let $X_{1} \subseteq X_{0} \times_{S} X_{0}$ denote the (locally) closed subspace of $X_{0} \times_{S} X_{0}$ representing the functor $\operatorname{Equ}\left(p_{1}^{*}(a), p_{2}^{*}(a)\right)$. Here $p_{i}: X_{0} \times_{S} X_{0} \rightarrow X_{0}$ indicates the $i$ th projection. Since $a$ is universal at every point of $X_{0}$, the morphism $p_{1}: X_{1} \rightarrow X_{0}$ is a local isomorphism. Moreover, $X_{1} \subseteq X_{0} \times_{S} X_{0}$ is an equivalence relation on $X_{0}$ (i.e., for every s-space $Z$, the set $\operatorname{Hom}_{S}\left(Z, X_{1}\right) \subseteq \operatorname{Hom}_{S}\left(Z, X_{0}\right) \times \operatorname{Hom}_{S}\left(Z, X_{0}\right) \cong \operatorname{Hom}_{S}\left(Z, X_{0} \times_{S} X_{0}\right)$ is an equivalence relation on $\operatorname{Hom}_{X}\left(Z, X_{0}\right)$ ). By (7.7) below, there is a quotient in the category of $S$-s-spaces, say $q: X_{0} \rightarrow Y$, which again is a local isomorphism. Since the sheaf axiom is satisfied, there is an $\eta \in F(Y)$ with $q^{*}(\eta)=a$. We claim that $(Y, \eta)$ represents $F$, i.e., for every $Z \in \mathrm{s}$-An $/ S$ the map

$$
\eta(Z): \operatorname{Hom}_{S}(Z, Y) \rightarrow F(Z), \quad f \mapsto f^{*}(\eta)
$$

is bijective. First we show that this map is injective. Consider $f_{1}, f_{2} \in$ $\operatorname{Hom}_{S}(Z, Y)$ with $f_{1}^{*}(\eta)=f_{2}^{*}(\eta)$. We must show that $f_{1}=f_{2}$. Obviously, we may assume that there are liftings $\tilde{f}_{i}: Z \rightarrow X_{0}$. By using the universal property of $X_{1}$ we obtain that the map $\left(\tilde{f}_{1}, \tilde{f}_{2}\right): Z \rightarrow X_{0} \times_{S} X_{0}$ factors through 
$X_{1}$. Hence $f_{1}=q \tilde{f}_{1}=q \tilde{f}_{2}=f_{2}$. Finally we must prove that $\eta(Z)$ is surjective. For this, take $b \in F(Z)$. Since $\eta(Z)$ is already shown to be injective it is sufficient to prove that every point $z \in Z$ has a neighborhood $U$ such that there exists an S-morphism $\phi: U \rightarrow X_{0}$ with $\phi^{*}(a)=b \mid U$. But this follows immediately from the construction, since $X_{0}$ contains the versal deformation of $b(z)$.

The following two lemmas have been used in the proof of (7.5).

(7.6) Lemma. Let $F$ be as in (7.5) and satisfy conditions (1)-(4). Let $T$ be a complex s-space over $S$ and $a \in F(T)$. Assume that $a$ is formally semiuniversal at some point $t_{0} \in T$. Then there is a neighborhood $U$ of $t_{0}$ in $T$ such that $a$ is universal at every point of $T$.

Proof. By (7.3), $a$ is even versal at $t_{0} \in T$. By openness of versality we may assume that $a$ is versal at every point of $T$. Let $T_{1} \subseteq T \times{ }_{S} T$ represent the functor $\operatorname{Equ}\left(p_{1}^{*}(a), p_{2}^{*}(a)\right)$, where $p_{i}$ is the $i$ th projection. Since $\left(t_{0}, t_{0}\right) \in T_{1}$ and $T_{1}$ is locally closed, we may even assume that $T_{1}$ is closed. Since $a$ is semiuniversal at $t_{0}$, we easily get that $T_{1}$ is the diagonal in a neighborhood of $\left(t_{0}, t_{0}\right)$ (check that $\xi:=p_{1}^{*}(a)\left|T_{1}=p_{2}^{*}(a)\right| T_{1}$ is semiuniversal at $\left.\left(t_{0}, t_{0}\right) \in T_{1} !\right)$. Hence, after shrinking $T$ as a neighborhood of $t_{0}$, we may suppose that $T_{1}$ is the diagonal of $T \times{ }_{S} T$. But then $a$ obviously is universal.

(7.7) Lemma. Let $X_{0}$ be a s-space over $S$ and $X_{1} \subseteq X_{0} \times_{S} X_{0}$ a locally closed (resp. closed) subspace. Assume that $X_{1}$ is an equivalence relation on $X_{0}$ and that the restriction of the first projection $p_{1}: X_{1} \rightarrow X_{0}$ is a local isomorphism. Then there is a not necessarily separated (resp. separated) s-space $Y$ together with a morphism $g: X_{0} \rightarrow Y$ such that $(Y, g)$ is the quotient of the equivalence relation given by $X_{1}$.

The proof is a very simple exercise. We remark that, similarly as in [Ki] or [Gra ${ }_{2}$, there should hold a much more general result.

In our final result we will give a criterion for a functor $F$ to have a coarse moduli space. Similar to the even case, we say that a functor

$$
F:(\mathrm{s}-\mathrm{An} / S)^{\circ} \rightarrow \text { sets }
$$

admits a coarse moduli space, say $M$, over $S$ if the following three conditions are satisfied.

(1) There is a functorial morphism

$$
\phi_{T}: F(T) \rightarrow \operatorname{Hom}_{S}(T, M), \quad T \in \mathrm{s}-\mathrm{An} / S .
$$

(2) If $T=\{t\}$ is a point then $\phi_{T}$ is even bijective.

(3) If $M^{\prime}$ is in s-An/S and

$$
\psi_{T}: F(T) \rightarrow \operatorname{Hom}_{S}\left(T, M^{\prime}\right), \quad T \in \mathrm{s}-\mathrm{An} / S,
$$

is a functorial morphism then there is a unique morphism $M \rightarrow M^{\prime}$ such that the diagram

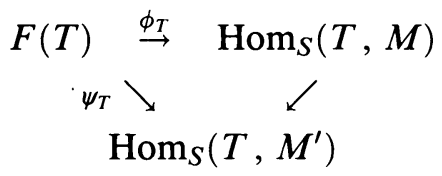

is commutative. 
If $F$ is a functor as above we can associate to $F$ its sheafification $\widetilde{F}$, i.e., $\widetilde{F}(T):=\Gamma\left(T,{ }_{T} \widetilde{F}\right)$, where ${ }_{T} \widetilde{F}$ denotes the sheaf associated to the presheaf $U \mapsto F(U)$ on $T$. With these notations, the next result follows immediately from the definitions.

(7.8) Proposition. If the sheaf $\widetilde{F}$ associated to $F$ is representable then $F$ has a coarse moduli space. More precisely, if $\widetilde{F}$ is represented by $M \in \mathrm{s}-\mathrm{An} / S$ then $M$ is a coarse moduli space for $F$.

\section{THE (L3)-CONDITION FOR FAMILIES OF COMPACT COMPLEX S-SPACES AND MODULES}

For the results in this section, it is convenient to consider the following abstract setting.

(8.1) Let $\wedge$ be a fixed analytic s-algebra and $p: \mathscr{F} \rightarrow s \mathscr{C}_{\wedge}$ a cofibered groupoid satisfying (S1), (S2), and $\mathrm{F}(\mathbb{C})=\left\{a_{0}\right\}$. A pair $(A, a), A \in s \mathscr{C}_{\wedge}, a \in \mathscr{F}(A)$, is called even versal if $a$ is even (i.e., $A$ is even) and if $(V)$ from (6.3) is satisfied for even $b, b^{\prime}$. We will say that $p$ satisfies (L3) if the associated functor $\bar{F}$ satisfies (L3) (see (1.12)). For $a \in \mathscr{F}(A)$ and a finite $A$-module $M$, the functors $\operatorname{Ex}(a, M), D_{a}(M)$, and $O_{a}(M)$ can be defined dually to (6.10), (6.5), and (6.15). We also need that there is an obstruction theory $\mathrm{Ob}_{a}(M)$, i.e., there is an injective functorial map $O_{a}(M) \stackrel{\mathrm{ob}}{\longrightarrow} \mathrm{Ob}_{a}(M)$, and $\mathrm{Ob}_{a}(-)$ is a functor on the category of finite $A$-modules into itself. For the next result we must also assume that for a morphism $a \rightarrow b$ in $\mathscr{F}$ over $A \rightarrow B$ in $s \mathscr{C}_{\wedge}$ and an $A$ linear map $M \rightarrow N$ of finite modules over $A$, resp. $B$, there is a functorial map $\mathrm{Ob}_{a}(M) \stackrel{\varphi}{\longrightarrow} \mathrm{Ob}_{b}(N)$ with the following property: If $A^{\prime}$, resp. $B^{\prime}$, is an extension of $A$, resp. $B$, by $M$, resp. $N$, such that there is a commutative diagram

$$
\begin{aligned}
& M \hookrightarrow A^{\prime} \rightarrow A \\
& \downarrow \downarrow \downarrow \\
& N \hookrightarrow B^{\prime} \rightarrow B
\end{aligned}
$$

then $\varphi$ maps ob $\left[A^{\prime}\right]$ onto ob $\left[B^{\prime}\right]$. If $\bar{A} \in s \widehat{\mathscr{C}}_{\wedge}, \bar{a} \in \widehat{\mathscr{F}}(\bar{A})$, and $\bar{M}$ is a finite $\bar{A}$-module then we set $\mathrm{Ob}_{a}(\bar{M}):=\lim \mathrm{Ob}_{\bar{a}_{n}}\left(\bar{M}_{n}\right)$, where the index $n$ means tensoring with $\bar{A} / \mathrm{m}_{\bar{A}}^{n+1}$. With these notations we will show

(8.2) Lemma. Assume that the following two conditions are satisfied.

(1) There exists an even versal deformation $(C, c)$ of $a_{0}$.

(2) Let $\bar{A} \in s \hat{\mathscr{C}}_{\wedge}$ and $\bar{A}=\lim _{\longrightarrow} A_{i}$ with $A_{i} \in s \mathscr{C}_{\wedge}$ and assume that $\bar{M}$ is a finite $\bar{A}$-module with $\bar{M}=\underline{\lim } M_{i}$, where $M_{i}$ is a finite $A_{i}$-module. Let $\bar{a} \in \widehat{\mathscr{F}}(\bar{A})$ and $a_{i} \in \mathscr{F}\left(A_{i}\right)$ with $\overrightarrow{a_{i}} \otimes A_{j}=a_{j}$ for $j \geq i$ and $\bar{a}=a_{i} \otimes \bar{A}$. Then the maps

(i) $\lim _{\longrightarrow} D_{a_{i}}\left(M_{i}\right) \rightarrow D_{\bar{a}}(\bar{M})$,

(ii) $\underset{\lim }{\longrightarrow} \mathrm{Ob}_{a_{i}}\left(M_{i}\right) \rightarrow \mathrm{Ob}_{a}(\bar{M})$

are surjective, resp. injective.

Then $p$ satisfies the (L3)-condition (see (1.12)). 
Proof. Assume that $\bar{B}=\lim _{\longrightarrow} B_{i}$ and $\bar{b} \in \widehat{\mathscr{F}}(\bar{B})$ is given. We will proceed by induction on the integer $\lambda:=\lambda(\bar{B}):=\min \left\{l \mid\left(\bar{B} \cdot \bar{B}_{\text {odd }}\right)^{l}=0\right\}$. If $\lambda=1$ then $\bar{B}$ is even and hence there is a map $C \stackrel{\varphi}{\rightarrow} \bar{B}$ with $\bar{b}=c \otimes_{C} \bar{B}$. Since $\varphi$ factors through some $B_{i}$, we obtain that $\bar{b}$ comes from $b_{i}:=c \otimes B_{i}$ and so we are done.

Now assume that $\lambda>0$ and set $\bar{M}:=\left(\bar{B}_{\text {odd }} \bar{B}\right)^{\lambda-1}, \bar{A}:=\bar{B} / \bar{M}$. We may suppose that $\bar{M}=\underset{\lim }{\longrightarrow} M_{i}$ with $M_{i} \subseteq B_{i}$. By applying the induction hypothesis we obtain $a_{i_{0}} \in \mathscr{F}\left(\overrightarrow{A_{i_{0}}}\right), A_{i_{0}}:=B_{i_{0}} / M_{i_{0}}$, such that $a_{i_{0}} \otimes \bar{A}=\bar{a}:=\bar{b} \otimes_{\bar{B}} \bar{A}$. Let $a_{i}:=a_{i_{0}} \otimes A_{i}$ for $i \geq i_{0}$. Because of our condition (2)(ii) above, we may assume that the image of $\left[B_{i_{0}}\right] \in T_{A_{i_{0}} / \wedge}^{1}\left(M_{i_{0}}\right)$ in $\mathrm{Ob}_{a_{i_{0}}}\left(M_{i_{0}}\right)$ is zero. Hence there is a $b_{i_{0}} \in \mathscr{F}\left(B_{i_{0}}\right)$ inducing $a_{i_{0}}$ in $\mathscr{F}\left(A_{i_{0}}\right)$. Let $\bar{b}_{i_{0}}$ be the object of $\widehat{\mathscr{F}}(\bar{B})$ induced by $b_{i_{0}}$. Then $\bar{b}_{i_{0}}$ and $\bar{b}$ differ by an element of $D_{\bar{a}}(\bar{M})$. Because of (i), we may assume that this element comes from an element of $D_{a_{i_{0}}}\left(M_{i_{0}}\right)$, and by changing $b_{i_{0}}$ by this element we get $\tilde{b}_{i_{0}} \in \mathscr{F}\left(B_{i_{0}}\right)$ which induces $\bar{b} \in \widehat{\mathscr{F}}(\bar{B})$.

As an application we will show

(8.3) Theorem. For deformations of compact complex s-spaces (see (6.4)(1)), the (L3)-condition is satisfied.

Proof. We will apply (8.2). By $\S 4$, there is an obstruction theory as described in (8.1). By (5.7), condition (2) of (8.2) is satisfied, and from (8.4) we get the existence of even versal deformations. Now (8.2) gives the desired result.

(8.4) Proposition. A compact complex s-space $X$ admits an even versal deformation.

Set $Y:=X_{\text {ev }}, \mathscr{M}:=\left(\mathscr{O}_{X}\right)_{1}$. We will first consider deformations of the pair $(Y, \mathscr{M})$ over even base spaces (see (8.5)). Then (8.4) will follow from (8.6).

(8.5) Lemma. There exists an (even) versal deformation of the pair $(Y, M)$.

Here by a deformation of $(Y, \mathscr{M})$ we mean a proper flat map $\mathscr{Y} \rightarrow S$ and

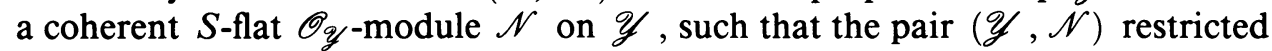
to the fiber over some point $s_{0} \in S$, is isomorphic to $(Y, \mathscr{M})$.

Proof of (8.5). Let

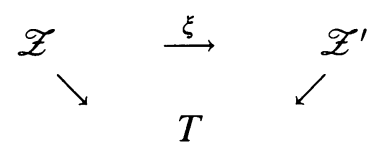

be the versal deformation of the holomorphic map $Y \rightarrow Y[\mathscr{M}]$ over the germ $\left(T, t_{0}\right)$ (see $\left.\left[\mathrm{F}_{1},(8.5)\right]\right)$. By [Do, Po], the morphism functor $M:=$ $\operatorname{Mor}_{T}\left(\mathscr{Z}^{\prime}, \mathscr{Z}\right)$ on the category of complex spaces over $T$, given by

$$
M\left(T^{\prime}\right):=\left\{f: \mathscr{Z}_{T^{\prime}}^{\prime} \rightarrow \mathscr{Z}_{T^{\prime}}: f \text { is a } T^{\prime} \text {-morphism }\right\},
$$

is representable by a complex space $M$ over $T$. Let $p \in M$ be the point associated to the canonical morphism $Y[\mathscr{M}] \rightarrow Y$. Denote by $\zeta: \mathscr{Z}_{M}^{\prime} \rightarrow \mathscr{Z}_{M}$ the universal morphism. There is a closed subgerm $\left(T_{1}, p\right) \hookrightarrow(M, p)$ such that $T_{1}$ is universal with the property that $\left(\zeta \circ \xi_{M}\right)_{T_{1}}=\mathrm{id}_{\mathscr{X}_{T_{1}}}$. This is easily seen by using the existence of the morphism space $\operatorname{Mor}_{M}\left(\mathscr{Z}_{M}, \mathscr{Z}_{M}\right)$. 
Now, over $T_{1}$, the sheaf $\mathscr{O}_{\mathscr{I}_{T_{1}}^{\prime}}$ is a direct sum $\mathscr{O}_{\mathscr{I}_{T_{1}}^{\prime}}=\mathscr{O}_{\mathscr{T}_{T_{1}}} \oplus \mathscr{N}_{1}, \mathscr{N}_{1}:=$ $\operatorname{ker}\left(\xi_{T_{1}}\right)$. The multiplication on $\mathscr{O}_{\mathscr{Z}_{T_{1}}}$ induces a map $\mathscr{N}_{1} \otimes \mathscr{N}_{1} \stackrel{\mu}{\rightarrow} \mathscr{N}_{1}$, and we denote by $\mathscr{K}$ the cokernel of $\mu$. By $\left[\mathrm{F}_{1}\right]$, there is a subgerm $(S, p) \subseteq\left(T_{1}, p\right)$ which is universal with the property that $\mathscr{K}_{S}$ is flat over $S$. Since $\mu$ is zero on the special fiber over $p, S$ is also the universal subspace with the property $\mu_{S}=0$. Now the reader may easily verify that the pair $\mathscr{Y}:=\mathscr{Z}_{S} \rightarrow S, \mathscr{N}:=$ $\mathscr{N}_{1 S}$ is the versal deformation of the pair $(Y, \mathscr{M})$.

(8.6) Lemma. Let $X, Y:=X_{\mathrm{ev}}, \mathscr{M}:=\mathscr{O}_{X_{1}}$ be as above, $\mathscr{Y} \rightarrow S$, and $\mathscr{N}$ be the versal deformation of the pair $(Y, \mathscr{M})$ (see (8.5)). Then there is a space $(T, 0) \rightarrow(S, 0)$ and a map

$$
\mathscr{N}_{T} \otimes \mathscr{N}_{T} \stackrel{\mu}{\rightarrow} \mathscr{O}_{Y_{T}}
$$

with the following properties:

(1) $\mu$ is anticommutative and $\mathscr{O}_{Y_{T}}$-linear.

(2) $\mathscr{O}_{X}:=\mathscr{O}_{\mathscr{Y}_{T}} \oplus \mathscr{N}_{T}$ is, together with the multiplication induced by $\mu$, an s-algebra.

(3) If $\left(T^{\prime}, t_{0}\right) \rightarrow\left(S, s_{0}\right)$ is any morphism such that there is a map

$$
\mathscr{N}_{T^{\prime}} \otimes \mathscr{N}_{T^{\prime}} \stackrel{\mu^{\prime}}{\rightarrow} \mathscr{O}_{\mathscr{F}_{T^{\prime}}}
$$

satisfying (1) and (2) then there is a unique S-morphism $\left(T^{\prime}, t_{0}\right) \stackrel{\Psi}{\rightarrow}\left(T, t_{0}\right)$ with $\psi^{*}(\mu)=\mu^{\prime}$, and $\psi^{*}\left(\mathscr{O}_{X}\right) \cong \mathscr{O}_{X^{\prime}}:=\mathscr{O}_{\mathscr{F}_{T^{\prime}}} \oplus \mathscr{N} \mid T^{\prime}$.

It is clear that (8.6) implies (8.4).

Proof of (8.6). Set $T_{1}:=\operatorname{Mor}_{S}\left(\bigwedge^{2} \mathscr{N}, \mathscr{O}_{\mathscr{Y}}\right)$ and let $\mu_{1}: \Lambda^{2} \mathscr{N}_{T_{1}} \rightarrow \mathscr{O}_{\mathscr{g} T_{1}}$ be the universal morphism. Observe that $\operatorname{Mor}_{S}\left(\Lambda^{2} \mathscr{N}, \mathscr{O}_{\mathscr{Y}}\right)$ exists as follows from Douady's Theorem or by applying $\left[F_{2},(3.2)\right]$. Now there are two maps deduced from $\mu_{1}$ :

$$
\mathscr{N}_{T_{1}} \otimes \mathscr{N}_{T_{1}} \otimes \mathscr{N}_{T_{1}} \underset{\beta}{\stackrel{\alpha}{\rightrightarrows}} \mathscr{N}_{T_{1}}
$$

The first one, $\alpha$, is obtained by applying $\mu_{1}$ to the first two factors and then multiplying with the third one, whereas $\beta$ maps $x \otimes y \otimes z$ to $x \cdot \mu_{1}(y \wedge z)$. Let $t_{0} \in T_{1}$ be the point in $T_{1}$ corresponding to the given map $\mathscr{M} \times \mathscr{M} \rightarrow \mathscr{O}_{Y}$ induced by the s-algebra structure on $\mathscr{O}_{X}=\mathscr{O}_{Y} \times \mathscr{M}$. By (8.7) there is a universal subgerm $\left(T, t_{0}\right) \subseteq\left(T_{1}, t_{0}\right)$ such that $\alpha_{T}=\beta_{T}$. Obviously, $\mu:=\mu_{T}$ has the required properties (1)-(3).

In the proof above we used the following result of Pourcin [Po] (see also $\left[\mathrm{F}_{2}\right.$, (3.3)]).

(8.7) Theorem. Let $f: X \rightarrow S$ be a morphism of (even!) complex spaces and $\mathscr{F}, \mathscr{G}$ be coherent $\mathscr{O}_{X}$-modules such that $\operatorname{supp}(\mathscr{F}) \cap \operatorname{supp}(\mathscr{G})$ is proper over $S$. Assume that $\mathscr{G}$ is flat over $S$ and that $f: \mathscr{F} \rightarrow \mathscr{G}$ is an $\mathscr{O}_{X}$-linear map. Then, for every $s_{0} \in S$, there exists a closed subgerm $\left(T, s_{0}\right) \subseteq\left(S, s_{0}\right)$ with the following properties:

(1) $f_{T}: \mathscr{F}_{T} \rightarrow \mathscr{G}_{T}$ is the zero map in a neighborhood of $s_{0}$.

(2) If $\left(S^{\prime}, s_{0}^{\prime}\right) \stackrel{\alpha}{\rightarrow}\left(S, s_{0}\right)$ is a morphism satisfying (1) then $\alpha\left(S^{\prime}\right) \subseteq T$ near $s_{0}$.

In the rest of this section we will show a similar result for modules. 
(8.8) Theorem. For deformations of modules (see (6.4)(2)), the (L3) condition is satisfied.

Proof. Again we will use (8.2). By the following Proposition (8.9), condition (1) in (8.2) is satisfied. Moreover, if $X \stackrel{f}{\rightarrow} Y$ is proper and $\mathscr{M} \in \operatorname{Coh}_{p, f}(X)$ then, for the groupoid described in (6.4)(2) and the object $a \in \mathscr{F}(Y)$ given by $\mathscr{M}$, the module $D_{a}(\mathscr{N})$ is easily seen to be isomorphic to $\operatorname{Ext}_{X}^{1}\left(\mathscr{M}, \mathscr{M} \otimes f^{*} \mathscr{N}\right)$ if $\mathscr{N} \in \operatorname{Coh}(Y)$. An obstruction theory is given by

$$
\mathrm{Ob}_{a}(\mathscr{N}):=\operatorname{Ext}_{X}^{2}\left(\mathscr{M}, \mathscr{M} \otimes f^{*}(\mathscr{N})\right)
$$

as can be seen as in the classical case (see, e.g., $\left[\mathrm{B}_{2},(8.3)\right]$ or $\left.\left[\mathrm{F}_{1},(4.12)\right]\right)$. Now (8.2) and (5.4) imply the result.

It remains to prove.

(8.9) Proposition. Let $X \stackrel{\pi}{\rightarrow} S$ be a morphism of complex s-spaces, $s_{0} \in S$ and $X_{0}=\pi^{-1}\left(s_{0}\right)$. If $\mathscr{M}$ is a coherent $\mathscr{O}_{X_{0}}$-module with compact support then $\mathscr{M}$ admits an even versal deformation (see (6.4)(2)).

Proof. We may assume that $S$ is even. If $X=X_{0}$, i.e., $S=\left\{s_{0}\right\}$, then the result follows from (8.10). In the general case, we consider first the even versal deformation of $\mathscr{M}$ on $X$ as in (8.10), i.e., there is a $T$-flat $\mathscr{O}_{X \times T}$-module $\mathscr{N}$ with proper support over an even space germ $\left(T, t_{0}\right)$ with $\mathscr{N}\left(t_{0}\right)=\mathscr{M}$, which is the even versal deformation of $\mathscr{M}$ in the sense of (8.10). Now consider $X \times T$ as space over $S \times T$ via $\pi \times 1_{T}$. Since $T \times S$ is even, we can apply [Fr] and obtain a subgerm $(\Sigma, 0) \subseteq\left(T \times S,\left(t_{0}, s_{0}\right)\right)$ such that $\mathscr{N}_{\Sigma}=\mathscr{N} \otimes \mathscr{O}_{\Sigma}$ is flat over $\Sigma$ and which is universal with this property. The reader may easily verify that $\mathscr{N}_{\Sigma}$ is the required even versal deformation of $\mathscr{M}$ over $X / S$.

In the proof of (8.9) we used

(8.10) Proposition. Let $X$ be a complex s-space and $\mathscr{M}$ a coherent $\mathscr{O}_{X}$-module with compact support. Then there exists an even versal deformation of $\mathscr{M}$.

Proof. By using the result of Siu-Trautmann [SIT] we obtain versal deformations, say $\mathscr{N}, \mathscr{N}_{0}$, and $\mathscr{N}_{1}$ of $\mathscr{M}, \mathscr{M}_{0}, \mathscr{M}_{1}$ over a suitable basis $T, T_{0}$, and $T_{1}$, respectively. Here we consider $\mathscr{M}, \mathscr{M}_{0}$, and $\mathscr{M}_{1}$ as $\mathscr{O}_{X_{\text {ev }}}$-modules. By using the representability of the morphism functor for modules it is easily seen that there is an (even) space germ $S$ over $T \times T_{0} \times T_{1}$ such that the module $\mathscr{N}_{S}$ has a "universal" decomposition $\mathscr{N}_{S} \cong \mathscr{N}_{0 S} \otimes \mathscr{N}_{1 S}$. Here $\mathscr{N}_{S}$ indicates the module on $X_{\text {ev }} \times S$ induced by $\mathscr{N}$ via the map $S \rightarrow T \times T_{0} \times T_{1} \rightarrow T$, similarly for $\mathscr{N}_{0 S}$ and $\mathscr{N}_{1 S}$. Now it remains to equip $\mathscr{N}_{S} \cong \mathscr{N}_{0 S} \oplus \mathscr{N}_{1 S}$ with an $\mathscr{O}_{X \times S}$-module structure (after suitable base change $S^{\prime} \rightarrow S$ ) inducing the given $\mathscr{O}_{X}$-structure on the special fiber. But this can be done with similar arguments as in the proof of (8.4).

\section{Applications}

In this section we will give several applications of the results of the preceding sections to moduli problems in supergeometry. We begin by showing that there is a versal deformation for modules. 
(9.1) Theorem. Let $f: \mathfrak{X} \rightarrow S$ be a morphism of s-spaces, $s_{0} \in S$, and $\mathscr{M}$ a coherent module on $X:=f^{-1}\left(s_{0}\right)$ with compact suppport. Then there exists a versal deformation of $\mathscr{M}$ (see (6.4)(2)).

Proof. By (6.14), there exists a formal deformation of $\mathscr{M}$ which is formally versal. By (8.8), the (L1)-condition is satisfied and hence there is even a convergent deformation of $\mathscr{M}$ which is formally versal (see (7.1)). By (7.3), this deformation of $\mathscr{M}$ is versal. Observe that the Iso-functor in (7.3) is representable as follows from (5.10).

We treat next the Hilbert moduli space. Let $f: X \rightarrow S$ be a morphism of s-spaces and let $\mathscr{F}$ be a coherent $\mathscr{O}_{X}$-module. Then we consider the Hilbertfunctor

$$
\text { Quat } \mathscr{F} / S:(\mathrm{s}-\mathrm{An} / S)^{\circ} \rightarrow \text { sets }
$$

which assigns to $T \rightarrow S$ the set of all quotients $\mathscr{G}$ of $\mathscr{F}_{T}$ which are proper and flat over $T$. In the case of complex spaces this is the Douady-functor, and by a result of Douady [Do] (resp. Pourcin [Po]) this functor is representable. Our next result shows that this is also true for s-spaces:

(9.2) Theorem. $\mathscr{Q}:=Q_{\text {Quat }} / S$ is representable by a complex s-space $Q$ over $S$.

Proof. We will show that $\mathscr{Q}$ satisfies conditions (1)-(4) of (7.5). The sheaf axiom for $\mathscr{Q}$ (see $(7.5)(2))$ is obvious. Openness of versality has been shown in (6.20), whereas (7.5)(3) is just (5.11); the Equ-functor in $(7.5)(3)$ is even represented by a closed subspace and so $Q$ will become separated if $S$ is. It remains to prove the existence of semiuniversal deformations. Consider $s_{0} \in S$ and a quotient $\mathscr{G}_{0}$ of $\mathscr{F}\left(s_{0}\right)$. By $(9.1), \mathscr{G}_{0}$ has a versal deformation, say $\mathscr{G}$, over some base space $\left(T, t_{0}\right) \rightarrow\left(S, s_{0}\right)$. Then it is easily seen that $\mathscr{G}_{M}$ is a versal deformation of $\mathscr{G}_{0}$ as a quotient of $\mathscr{F}\left(s_{0}\right)$, where

$$
M:=\operatorname{Mor}_{T}\left(\mathscr{F}_{T}, \mathscr{G}\right)
$$

(see (5.10)). Here $M$ is regarded as a germ around the point belonging to the natural projection $\mathscr{F}\left(s_{0}\right) \rightarrow \mathscr{G}_{0}$.

(9.3) Corollary. Let $\mathfrak{X} \rightarrow S, \mathfrak{Y} \rightarrow S$ be morphism of complex s-space and assume that $\mathfrak{X} \rightarrow S$ is proper and flat. Then the functor

$$
\mathscr{M}_{\text {or } S}(\mathfrak{X}, \mathfrak{Y}):(\mathrm{s}-\mathrm{An} / S)^{\circ} \rightarrow \text { sets, }
$$

where $\mathscr{M}_{S}(\mathfrak{X}, \mathfrak{Y})(T)$ is the set of all T-morphisms $\mathfrak{X}_{T} \rightarrow \mathfrak{Y}_{T}$, can be represented by a (separated) complex s-space $\operatorname{Mor}_{S}(\mathfrak{X}, \mathfrak{Y})$ over $S$.

Proof. Let $\mathscr{H}$ denote the quotient functor for the sheaf $\mathscr{F}:=\mathscr{O}_{\mathfrak{X} \times s \mathfrak{Y}}$ on $\mathfrak{X} \times S$ $\mathfrak{Y}$ (see (9.2)). By identifying a morphism with its graph we see that $\mathscr{M}_{\text {or } S}(\mathfrak{X}, Y)$ may be regarded as a subfunctor of $\mathscr{H}$. Let $H$ represent $\mathscr{H}$ and denote by $\Gamma \subseteq \mathfrak{X} \times{ }_{S} \mathfrak{Y} \times{ }_{S} H$ the universal subspace. Let $p: \Gamma \rightarrow \mathfrak{X} \times{ }_{S} H$ indicate the projection. We must show that there exists a maximal subspace $M \subseteq H$ for which $p_{M}: \Gamma_{M} \rightarrow \mathfrak{X} \times_{S} M$ is an isomorphism and which is universal with this property. Clearly, such an $M$ would represent $\mathscr{M}_{\text {or }}(\mathfrak{X}, \mathfrak{Y})$. Hence (9.3) will follow from

(9.4) Lemma. Let $f: X \rightarrow Y$ be a morphism of complex s-spaces over $S$. Assume that $X \rightarrow S$ is proper and flat and that $Y \rightarrow S$ is proper. Then the 
functor

$$
F:(\mathrm{s}-\mathrm{An} / S)^{\circ} \rightarrow \text { sets }
$$

given by

$$
F(T):= \begin{cases}\{1\} & \text { if } f_{T} \text { is an isomorphism, } \\ \varnothing & \text { otherwise, }\end{cases}
$$

is represented by an open subset of $S$.

The proof of (9.4) is an easy exercise.

(9.5) Theorem. Let $X_{0}$ be a compact complex s-space. Then $X_{0}$ admits $a$ versal deformation.

Proof. We will show that conditions (1)-(3) of (7.4) are fulfilled. For the (L1) condition, this follows from (8.3). The Schlessinger conditions are obviously true in this case (see (6.7)). Finally, that $(7.4)(2)$ is satisfied follows from (9.3) and (9.4).

(9.6) Let $f: X \rightarrow S$ be a proper flat morphism of complex s-spaces. We will assume that $f$ is cohomologically flat in dimension 0 , i.e., for every $s \in S$, the natural map

$$
f_{*}\left(\mathscr{O}_{X}\right)(s) \rightarrow f_{*}\left(\mathscr{O}_{X(s)}\right)
$$

is surjective. Let $\mathscr{M}$ be a coherent $\mathscr{O}_{X}$-module which is simple over $S$. By this we mean that $\mathscr{M}$ is $S$-flat and that the natural map

$$
f_{*}\left(\mathscr{O}_{X(s)}\right) \rightarrow f_{*}\left(\mathscr{H} \circ m_{X}(\mathscr{M}, \mathscr{M}(s))\right)
$$

is bijective for every $s \in S$.

The next result shows that these notations behave well under base change.

(9.7) Lemma. Let $f: X \rightarrow S$ and $\mathscr{M}$ be as in (9.6). Let $T$ be a complex s-space over $S$. Then the following hold.

(0) $f_{*}\left(\mathscr{O}_{X}\right)$ is locally free as an $\mathscr{O}_{S}$-module.

(1) $f_{T}: X_{T} \rightarrow T$ is cohomologically flat in dimension 0 and $\mathscr{M}_{T}$ is simple over $T$.

(2) Every $\mathscr{L} \in \operatorname{Pic}(X)$ is simple over $S$.

(3) For every coherent $\mathscr{O}_{S}$-module $\mathscr{N}$, the natural map

$$
\alpha(\mathscr{N}): f_{*}\left(\mathscr{O}_{X}\right) \otimes \mathscr{N} \rightarrow f_{*}\left(\mathscr{H} o m_{X}\left(\mathscr{M}, \mathscr{M} \otimes f^{*}(\mathscr{N})\right)\right)
$$

is bijective.

Observe that (3) in particular implies that $f_{*}\left(\mathscr{O}_{X}\right) \otimes \mathscr{N}$ and $f_{*} f^{*}(\mathscr{N})$ are naturally isomorphic.

Proof. Assume first that the map $(*)$ in $(9.6)$ is only surjective. Let $\mathscr{K}:=$ $f_{\#}(\mathscr{M})$ be as in (5.8) such that

$$
f_{*}\left(\mathscr{H} \circ m_{X}\left(\mathscr{M}, \mathscr{M} \otimes f^{*}(\mathscr{N})\right)\right) \cong \mathscr{H} o m_{S}(\mathscr{K}, \mathscr{N})
$$

for every $\mathscr{N} \in \operatorname{Coh}(S)$. Since $(*)$ is onto, the natural map $\mathscr{H} o m_{S}\left(\mathscr{K}, \mathscr{O}_{S}\right) \rightarrow$ $\mathscr{H} \operatorname{om}_{S}\left(\mathscr{K}, \mathscr{O}_{S}(s)\right)$ is surjective for every point $s \in S$. This implies that $\mathscr{K}$ is locally free, and so $\beta(\mathscr{N}): f_{*}\left(\mathscr{H} o m_{X}(\mathscr{M}, \mathscr{M})\right) \otimes \mathscr{N} \rightarrow f_{*}\left(\mathscr{H} o m_{X}(\mathscr{M}, \mathscr{M} \otimes\right.$ $\mathscr{N}))$ is bijective for every $\mathscr{N} \in \operatorname{Coh}(\mathscr{N})$. In particular, $f_{*}\left(\mathscr{O}_{X}\right) \otimes \mathscr{N} \cong f_{*} f^{*}(\mathscr{N})$. Therefore the functor $\mathscr{N} \mapsto f_{*}\left(\mathscr{O}_{X}\right) \otimes \mathscr{N}$ is left exact since this is true for $f_{*}$ 
and $f^{*}$. This proves (0). Assertions (1) and (2) are trivial. For the proof of (3), assume that $\mathscr{M}$ is simple over $S$. Then the natural map

$$
f_{*}\left(\mathscr{O}_{X}\right)(s) \rightarrow f_{*} \mathscr{H} \operatorname{om}_{X}(\mathscr{M}, \mathscr{M})(s)
$$

is bijective for every $s \in S$ as follows from $(*)$ and the bijectivity of $\beta(\mathcal{N})$. Since $f_{*}\left(\mathscr{O}_{X}\right)$ is locally free as an $\mathscr{O}_{S}$-module, this easily implies that

$$
f_{*}\left(\mathscr{O}_{X}\right) \widetilde{\sim} f_{*} \mathscr{H} \operatorname{om}_{X}(\mathscr{M}, \mathscr{M}) \text {. }
$$

This and the bijectivity of $\beta(\mathscr{N})$ yield (3).

Let $f: X \rightarrow S$ be cohomologically flat in dimension 0 . For a space $T \in$ s-An $/ S$, let $\mathscr{S}_{i m \mu_{X / S}}(T)$ denote the set of all coherent $\mathscr{O}_{X_{T}}$-modules $\mathscr{M}$ which are simple over $T$. Clearly,

$$
\operatorname{Sim}_{X / S}:(\mathrm{s}-\mathrm{An} / S)^{0} \rightarrow \text { sets }
$$

is a functor.

(9.8) Theorem. Let $f: X \rightarrow S$ be as above. Then there is a coarse moduli space $\operatorname{Simp}_{X / S}$ for $\mathscr{S i m}_{X / S}$.

We remark that, in general, $\operatorname{Simp}_{X / S}$ is not separated.

Proof. By (7.8) it is sufficient to prove that the sheaf $\mathscr{S}_{i m} \tilde{\sim}_{X / S}$ associated to

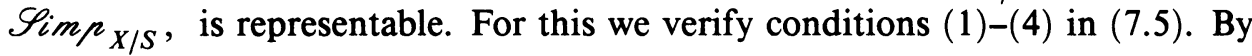
(9.1), condition (1) is satisfied. By (6.20), (4) is satisfied. Finally, (2) holds trivially and (3) follows from the subsequent Proposition (9.9).

(9.9) Proposition. Let $f: X \rightarrow S$ be as in (9.6) and assume that $\mathscr{F}, \mathscr{G}$ are coherent $\mathscr{O}_{X}$-modules which are simple over $S$. Then the functor

$$
F:(\mathrm{s}-\mathrm{An} / S)^{\circ} \rightarrow \text { sets },
$$

with

$$
F(T)= \begin{cases}\{1\} & \text { if } \mathscr{G}_{T} \text { and } \mathscr{F}_{T} \text { are locally in } T \text { isomorphic, } \\ \varnothing & \text { else, }\end{cases}
$$

is representable by a locally closed subspace of $S$.

Proof. By (5.8) there are sheaves $\mathscr{L}, \mathscr{K}$ on $S$ such that

$$
\begin{gathered}
f_{*} \mathscr{H} \operatorname{om}_{X}\left(\mathscr{F}, \mathscr{G} \otimes f^{*}(\mathscr{N})\right) \cong \mathscr{H} \operatorname{om}_{S}(\mathscr{L}, \mathscr{N}), \\
f_{*} \mathscr{H} \operatorname{om}_{X}\left(\mathscr{G}, \mathscr{F} \otimes f^{*}(\mathscr{N})\right) \cong \mathscr{H} \operatorname{om}_{S}(\mathscr{K}, \mathscr{N}),
\end{gathered}
$$

and $\mathscr{L}$ and $\mathscr{K}$ are compatible with arbitrary base change $T \rightarrow S$. Because of (9.7),

$$
f_{*} \mathscr{H} \operatorname{om}_{X}\left(\mathscr{F}, \mathscr{F} \otimes f^{*}(\mathscr{N})\right) \cong f_{*}\left(\mathscr{O}_{X}\right) \otimes \mathscr{N}=\mathscr{H} \operatorname{om}_{S}\left(f_{*}\left(\mathscr{O}_{X}\right)^{v}, \mathscr{N}\right),
$$

since $f_{*}\left(\mathscr{O}_{X}\right)$ is locally free. Hence, if $\mathscr{G}_{T}$ and $\mathscr{F}_{T}$ are locally isomorphic in $T$, then $\mathscr{L}_{T}$ and $\mathscr{K}_{T}$ are locally free on $T$ of the same rank as $f_{T *}\left(\mathscr{O}_{X_{T}}\right)$. Therefore, by using the following Lemma (9.10), we may assume that $\mathscr{L}, \mathscr{K}$ are already locally free of the same rank as $f_{*}\left(\mathscr{O}_{X}\right)$. In this case, the sheaves $f_{*} \mathscr{H} \operatorname{om}_{X}(\mathscr{F}, \mathscr{G})$ and $f_{*} \mathscr{H} \operatorname{om}_{X}(\mathscr{G}, \mathscr{F})$ are compatible with base change $T \stackrel{g}{\rightarrow}$ $S$, i.e.,

$$
f_{T_{*}}\left(\mathscr{H} \circ m_{X_{T}}\left(\mathscr{F}_{T}, \mathscr{G}_{T}\right)\right) \cong g^{*} f_{*} \mathscr{H} o m_{X}(\mathscr{F}, \mathscr{G}),
$$


similarly for $f_{*} \mathscr{H} o m_{X}(\mathscr{G}, \mathscr{F})$. Let $\mathscr{C}$, resp. $\mathscr{C}^{\prime}$, be the cokernel of the composition pairings

$$
\begin{gathered}
f_{*}\left(\mathscr{H} \operatorname{om}_{X}(\mathscr{F}, \mathscr{G})\right) \otimes f_{*}\left(\mathscr{H} \operatorname{om}_{X}(\mathscr{G}, \mathscr{F})\right) \rightarrow f_{*}\left(\mathscr{H} \operatorname{om}_{X}(\mathscr{F}, \mathscr{F})\right)=f_{*}\left(\mathscr{O}_{X}\right), \\
f_{*}\left(\mathscr{H} \operatorname{om}_{X}(\mathscr{G}, \mathscr{F})\right) \otimes f_{*}\left(\mathscr{H} \operatorname{om}_{X}(\mathscr{F}, \mathscr{G})\right) \rightarrow f_{*}\left(\mathscr{H} \operatorname{om}_{X}(\mathscr{G}, \mathscr{G})\right)=f_{*}\left(\mathscr{O}_{X}\right) .
\end{gathered}
$$

We claim that $F$ is represented by the open subspace $S \backslash \operatorname{supp}\left(\mathscr{C} \oplus \mathscr{C}^{\prime}\right)$. Since the composition pairings are compatible with base change $T \rightarrow S$, it is sufficient to prove that the surjectivity of the pairings above imply that $\mathscr{F}$ and $\mathscr{G}$ are (locally in $S$ ) isomorphic. But this is an easy consequence of (9.11).

In the proof of (9.9) we have used the following two lemmas.

(9.10) Lemma. Let $S$ be a complex s-space and $\mathscr{L} \in \operatorname{Coh}(S)$. For a fixed $r \in \mathbb{N}$, consider the set-valued functor on $\mathrm{s}-\mathrm{An} / S$ given by

$$
G(T)= \begin{cases}\{1\} & \text { if } \mathscr{L}_{T} \text { is locally free of rank } r, \\ \varnothing & \text { otherwise. }\end{cases}
$$

Then $G$ is representable by a locally closed subspace of $S$.

Proof. For $s \in S$ set $\mu_{s}(\mathscr{L}):=\operatorname{dim}_{\mathbb{C}}\left(\mathscr{L}_{s} / \mathfrak{m}_{s} \mathscr{L}_{s}\right)$, where $\mathfrak{m}_{s} \subseteq \mathscr{O}_{S, s}$ is the maximal ideal. The subset of all $s \in S$ with $\mu_{s}(\mathscr{L}) \leq r$ is easily seen to be an open subset, say $U$, of $S$. For every $s \in U$, there is a presentation

$$
\mathscr{F} \underset{\mathfrak{a}}{\rightarrow} \mathscr{G} \rightarrow \mathscr{L} \rightarrow 0
$$

on some neighborhood $V$ of $s$, where $\mathscr{F}, \mathscr{G}$ are free $\mathscr{O}_{V}$-modules and $\operatorname{rank}(\mathscr{G})$ $=r$. The closed suspace $A_{V}$ defined by the entries of $\mathfrak{a}$ is obviously independent of the presentation. Hence the closed subspaces $A_{V}$ define a closed subspace $A$ of $U$, and the reader may easily verify that $G$ is represented by $A$.

(9.11) Lemma. Let $X$ be a complex s-space. Let $\mathscr{F}, \mathscr{G}$ be coherent $\mathscr{O}_{X^{-}}$ modules and assume that there are morphisms

$$
\alpha, \beta: \mathscr{F} \rightarrow \mathscr{G} ; \quad \gamma, \delta: \mathscr{G} \rightarrow \mathscr{F}
$$

such that $\gamma \circ \alpha=\mathrm{id}_{\mathscr{F}}, \beta \circ \delta=\mathrm{id}_{\mathscr{G}}$. The $\alpha, \beta, \gamma, \delta$ are isomorphisms.

Proof. Let $x \in X$ be fixed and consider $F:=\mathscr{F}_{x}$ and $G:=\mathscr{G}_{x}$. Let $\alpha, \beta, \gamma, \delta$ also denote the morphisms on $F$, resp. $G$, associated to the given ones. By our assumption, $F$ is a direct summand of $G$ and $G$ is a direct summand of $F$. Therefore the lengths of the modules $F / \mathfrak{m}^{n+1} F$ and $G / \mathfrak{m}^{n+1} G$ are equal, where $\mathfrak{m} \subseteq \mathscr{O}_{X, x}$ denotes the maximal ideal. Hence $\alpha, \beta, \gamma, \delta$ are isomorphisms modulo $\mathrm{m}^{n+1}$ which easily yields the lemma.

As a simple application, we prove that the Picard-functor is representable in certain cases:

(9.12) Theorem. Let $f: X \rightarrow S$ be a proper flat morphism which is cohomologically flat in dimension 0 . Then the Picard-functor

$$
\mathscr{P}_{i \mathrm{C} / S}:(\mathrm{s}-\mathrm{An} \mid S)^{\circ} \rightarrow \text { sets },
$$

given by $\mathscr{P}_{i c / S}(T)=\Gamma\left(T, R^{1} f_{T *}\left(\mathscr{O}_{X_{T}, \mathrm{ev}}^{*}\right)\right)$, is representable by a (not necessarily separated) complex s-space over $S$.

Proof. With the notation as in the proof of (9.8), the functor $\mathscr{P}_{i C_{X / S}}$ is a

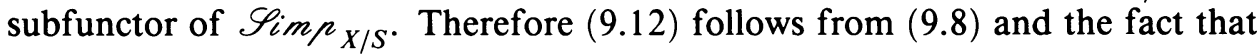


the set of points of $\operatorname{Simp}_{X / S}$ given by the locally free modules of rank 1 is an open subset in $\operatorname{Simp}_{X / S}$.

\section{REFERENCES}

[A $\left.A_{1}\right]$ M. Artin, On the solutions of analytic equations, Invent. Math. 5 (1968), 277-291.

[A $\left.\mathrm{A}_{2}\right]$ M. Artin, Algebraization of formal moduli. I, Global Analysis, University of Tokyo Press, 1969, pp. 21-71.

[A $\left.\mathrm{A}_{3}\right]$ M. Artin, Versal deformations and algebraic stacks, Invent. Math. 27 (1974), 165-189.

[Be] F. A. Berezin, Introduction to superanalysis (A. A. Kirilov, ed.), Reidel, 1987.

[Bi $\left.{ }_{1}\right] \quad J$. Bingener, Über formale komplexe Räume, Manuscripta Math. 24 (1978), 253-293.

$\left[\mathrm{Bi}_{2}\right] \quad$, Darstellbarkeitskriterien für analytische Funktoren, Ann. Sci. École Norm. Sup. (4) 13 (1980), 317-347.

[ $\left.\mathrm{Bi}_{3}\right] \quad-$ Offenheit der Versalität in der analytischen Geometrie, Math. Z. 173 (1980), 241-289.

[Bi-Ko] J. Bingener and S. Kosarew, Lokale Modulräume in der analytischen Geometrie, Vieweg, Braunschweig.

[De] P. Deligne, Letter to D. Sundararaman, April 7, 1987.

[Do] A. Douady, Le problème des modules pour les sous espaces analytiques compacts d'un espace analytique donne, Ann. Inst. Fourier (Grenoble) 16 (1966), 1-95.

[ $\left.\mathrm{F}_{1}\right] \quad$ H. Flenner, Deformationen holomorpher Abbildungen, Habilitationsschrift, Osnabrück, 1978.

$\left[\mathrm{F}_{2}\right]$ _ Eine Bemerkung über relative Ext-Garben, Math. Ann. 258 (1981), 175-182.

[ $\left.\mathrm{F}_{3}\right] \quad-$ Ein Kriterium für die Offenheit der Versalität, Math. Z. 178 (1981), 449-473.

[Fr] J. Frisch, Aplatissement en géomètrie analytique, Ann Sci. École Norm. Sup. (4), 1 (1968), 305-312.

[Gra ${ }_{1}$ H. Grauert, Der Satz von Kuranishi für kompakte komplexe Räume, Invent. Math. 25 (1974), 107-142.

[Gra $\left.{ }_{2}\right] \ldots$, On meromorphic equivalence relations (Proc. Conf. in honor of W. Stoll), Aspects of Math. E9 (1986), 115-147.

[Gro] A. Grothendieck, Techniques de construction en géométrie analytique. I-X, Séminaire Car$\tan , 1960,1962$.

[Ha] R. Hartshorne, Residues and duality, Lecture Notes in Math., vol. 20, Springer-Verlag, Berlin-Heidelberg-New York, 1966.

[Ki] R. Kiehl, Äquivalenzrelationen in analytischen Räumen, Math Z. 105 (1968), 1-20.

[Ko-Ok] S. Kosarew and C. Okonek, Global moduli spaces and simple holomorphic bundles, Mathematica Gottingensis, Heft 10, 1987.

[Ku] M. Kuranishi, New proof for the existence of locally complete families of complex structures, Proc. Conf. Complex Analysis (Minneapolis 1964), Springer-Verlag, 1965, pp. 142-154.

[L] D. A. Leites, Introduction to the theory of supermanifolds, Russian Math. Surveys 35 (1980), $1-64$.

[Ma $\left.{ }_{1}\right]$ Yu. I. Manin, New dimensions in geometry, Lecture Notes in Math., vol. 1111, SpringerVerlag, Berlin and New York, 1985, pp. 59-99.

$\left[\mathrm{Ma}_{2}\right]+$, Gauge field theory and complex geometry, Grundlehren der Math., 289, SpringerVerlag, 1988.

[Pa] V. P. Palamodov, Deformations of complex spaces, Russian Math. Surveys 31 (1984), 119171.

[Po] G. Pourcin, Théorèmes de Douady au-dessus de S, Ann. Scuola Norm. Sup. Pisa C. Sci. (4) 23 (1969), 451-459.

[Ri] R. S. Rim, Formal deformation theory, Groupes de Monodromie en Géométrie Algébrique (SGA7I), expose VI, Lecture Notes in Math., vol. 288, Springer-Verlag, Berlin and New York, 1972. 
[RR] J. P. Ramis and G. Ruget, Complexe dualisant et théorèmes de dualité en géométrie analytique complexe, Inst. Hautes Études Sci. Publ. Math. 38 (1971), 77-91.

[Sch] M. Schlessinger, Functors of Artin rings, Trans. Amer. Math. Soc. 130 (1968), 208-222.

[Schu ${ }_{1}$ ] H. W. Schuster, Formale Deformationstheorien, Habilitationsschrift, München, 1971.

[Schu $\left.{ }_{2}\right] \ldots$, Infinitesimale Erweiterungen komplexer Räume, Comment. Math. Helv. 45 (1970), 265-286.

[SIT] Y. T. Siu and G. Trautmann, Deformations of coherent analytic sheaves with compact supports, Mem. Amer. Math. Soc., No. 238 (1981).

[SS] G. Scheja and U. Storch, Differentielle Eigenschaften der Lokalisierungen analytischer Algebren, Math. Ann. 197 (1972), 137-170.

[W $\mathrm{W}_{1}$ A. Yu. Weintrob, Deformations of complex structures on supermanifolds, Functional Anal. Appl. 18 (1984), 59-60.

[W $\left.\mathrm{W}_{2}\right] \quad$, Deformations of complex structures on supermanifolds, Reports of the Dept. of Math. Univ. Stockholm, 1988.

Mathematisches Institut der Georg-August-Universität, Bunsenstrasse 3-5, D-3400 GötTINGEN, Germany

Department of Mathematics, University of California at San Diego, la Jolla, CaliFORNIA 92093

Current address: Department of Mathematics, Howard University, Washington, D.C. 20059 